\title{
JOURNAL OF THE BRAZILIAN SOCIETY OF MECHANICAL SCIENCES AND ENGINEERING
}

PUBLISHER: SPRINGER

IMPACT FACTOR: 1.235

ISSN: 1678-5878 (PRINT VERSION)

ISSN: 1806-3691 (ELECTRONIC VERSION)

Accepted January $28^{\text {th }} 2018$

\section{RADIATIVE AND MAGNETOHYDRODYNAMICS FLOW OF THIRD GRADE VISCOELASTIC FLUID PAST AN ISOTHERMAL INVERTED CONE IN THE PRESENCE OF HEAT GENERATION/ABSORPTION}

\author{
S. Abdul Gaffar ${ }^{1 *}$, V. Ramachandra Prasad², O. Anwar Bég ${ }^{3}$, \\ Md. H. Hidayathullah Khan ${ }^{4}$ and K. Venkatadri ${ }^{4}$ \\ ${ }^{1}$ Department of Mathematics, Salalah College of Technology, Salalah, Oman \\ ${ }^{2}$ Department of Mathematics, School of Advanced Sciences, VIT University, Vellore - 632014, Tamil Nadu, India. \\ ${ }^{3}$ Fluid Mechanics, Aeronautical and Mechanical Engineering Department, University of Salford, Manchester, M5, 4WT, UK. \\ ${ }^{4}$ Department of Mathematics, Madanapalle Institute of Technology and Science, Madanapalle-517325, India
}

\begin{abstract}
A mathematical analysis is presented to investigate the nonlinear, isothermal, steady-state, free convection boundary layer flow of an incompressible third grade viscoelastic fluid past an isothermal inverted cone in the presence of magnetohydrodynamic, thermal radiation and heat generation/absorption. The transformed conservation equations for linear momentum, heat and mass are solved numerically subject to the realistic boundary conditions using the second-order accurate implicit finite-difference Keller Box Method. The numerical code is validated with previous studies. Detailed interpretation of the computations is included. The present simulations are of interest in chemical engineering systems and solvent and low-density polymer materials processing.
\end{abstract}

Keywords: Viscoelastic fluid; third grade fluid parameter; solvent processing; skin friction; magnetohydrodynamics; thermal radiation.

*Corresponding author: abdulsgaffar0905@gmail.com

\section{NOMENCLATURE}

$A \quad$ half angle of the cone

$B_{0} \quad$ externally imposed radial magnetic field 
C concentration

$C_{f} \quad$ skin friction coefficient

$c_{p} \quad$ specific heat

$D_{m} \quad$ mass (species) diffusivity

$F \quad$ radiation parameter

$f \quad$ dimensionless stream function

$G r_{x} \quad$ local Grashof number

$g \quad$ acceleration due to gravity

$K \quad$ thermal diffusivity

$k \quad$ thermal conductivity of the fluid

$M \quad$ magnetic parameter

$N \quad$ buoyancy ratio parameter

$\mathrm{Nu} \quad$ local Nusselt number

$\mathrm{Pr} \quad$ Prandtl number

$q_{r} \quad$ radiative heat flux

$r \quad$ local radius of the truncated cone

Sc $\quad$ Schmidt number

Sh local Sherwood number

$T \quad$ fluid temperature

$u, v \quad$ dimensionless velocity components along the $x$ - and $y$-directions, respectively

V velocity vector

$x \quad$ stream wise coordinate

$y \quad$ transverse coordinate

\section{Greek Symbols}

$\alpha \quad$ thermal diffusivity

$\beta \quad$ coefficient of thermal expansion

$\beta^{*} \quad$ coefficient of concentration expansion

$\varepsilon_{l} \quad$ first viscoelastic material fluid parameter

$\varepsilon_{2} \quad$ second viscoelastic material fluid parameter

$\beta_{3} \quad$ third grade material parameter

$v \quad$ kinematic viscosity

$\rho \quad$ fluid density

$\mu \quad$ Newtonian dynamic viscosity 
$\eta \quad$ dimensionless radial coordinate

$\theta$ dimensionless temperature

$\phi \quad$ dimensionless concentration

$\Delta \quad$ heat generation/absorption parameter

$\varphi \quad$ third grade dimensionless viscoelastic fluid parameter

$\sigma^{*} \quad$ Stefan-Boltzmann constant

$\xi \quad$ dimensionless tangential coordinate

$\psi \quad$ dimensionless stream function

\section{Subscripts}

w surface conditions on cone (wall)

$\infty \quad$ free stream conditions

\section{INTRODUCTION}

Non-Newtonian fluid dynamics continues to grow due to the increasing applications in many industries such as china clay, coal in water, sewage sludge, oil-water emulsions, gas-liquid dispersions, coal-oil slurries, detergent and paint production, smart coating and suspension fabrication, pharmacology, cosmetic creams, physiological transport processes (blood, bile and synovial fluid), slurry conveyance, polymer synthesis and food processing. The mathematical models in non-Newtonian fluids are more complicated and relate the shear stresses to the velocity field [1]. Few non-Newtonian transport modeling include Casson non-Newtonian fluids [2], oblique micropolar stagnation flows [3], Walter's viscoelastic flows [4], Jeffrey's viscoelastic boundary layers [5], magnetized Williamson fluids [6], nanofluid transport from a sphere [7], Maxwell fluids [8] Eyring-Powell fluid [9], Tangent Hyperbolic fluid [10] and Jeffery Nano fluid [11-12].

Most non-Newtonian models involve some form of modification to the momentum conservation equations (Newton's second law). Several fluid models have however emerged as strong candidates in successfully mimicking actual non-Newtonian characteristics. Among these, the differential type fluid models have proved popular. The simplest subclass of these viscoelastic models is the second grade fluid, which describes the normal stress differences but cannot predict shear thinning/thickening phenomena. However, the third-grade fluid model is capable of predicting both normal stress and shear thinning/thickening phenomena. Many researchers have examined the flows of third-grade fluids for various scenarios, usually with a mathematical emphasis and very little if any, physical understanding or interpretation of the 
solutions. These studies are of very limited value to engineers working in complex (polymeric) fluid mechanics industries. For instance, Sahoo [13] investigated the flow and heat transfer of third grade fluid from an exponentially stretching sheet with partial slip boundary conditions. Aziz and Aziz [14] studied the magnetohydrodynamic flow of a third grade fluid in porous media with wall mass flux effects. Hayat et al. [15] analyzed axisymmetric flow of a magnetized third grade fluid between stretching sheets with heat transfer. Melting heat transfer in the stagnation-point flow of third grade fluid from an extending sheet with viscous dissipation was addressed by Hayat et al. [16] using the semi-analytical homotopy analysis method. A theoretical simulation of hydromagnetic axisymmetric flow of third grade fluid induced by a stretching cylinder was presented by Hayat et al. [17]. Samuel et al. [18] considered thermodynamic aspects of hydromagnetic third grade fluid flow in a porous media channel. Abdul hameed et al. [19] computed solutions for transient third-grade flow caused by the periodic motion of an infinite wall with transpiration. Rashidi et al. [20] conducted an entropy generation minimization analysis of convective magnetic flow of third grade non-Newtonian fluid from a stretching sheet. Again these studies did even not attempt to evaluate the physics of third grade fluid effects making them of minimal interest from an engineering perspective.

The influence of magnetic field has attracted the interest of researchers due to its applications in geophysics, astrophysics and many engineering problems like cooling of nuclear reactors, boundary layer control in aerodynamics and cooling towers. Aracely Lopez et al. [21] investigated numerically the heat transfer and entropy generation in a magnetohydrodynamic flow of nanofluid through a porous vertical microchannel with nonlinear Radiative heat flux using runge-Kutta integration method and shooting technique. Rashad [22] studied the magnetohydrodynamic mixed convection flow of Cobalt-kerosene Ferro fluid adjacent to a nonisothermal wedge under the influence of thermal radiation and partial slip using Thomas algorithm. Hayat et al. [23] presented mathematical analysis of magnetohydrodynamics threedimensional nonlinear convective flow of Maxwell nanofluid towards a stretching surface in the presence of thermal radiation, heat generation/absorption and heat flux. Hayat et al. [24] presented the convection flow of viscous fluid by a curved stretching sheet in the presence of uniform magnetic field, thermal radiation and chemical reaction. Jalilpour et al. [25] investigated the theoretical study of steady stagnation point flow with heat transfer of nanofluid towards a stretching surface in the presence of magnetohydrodynamics and thermal radiation using RungeKutta method. Dogonchi et al. [26] analyzed the unsteady squeezing flow and heat transfer of MHD nanofluid between the infinite parallel plates with thermal radiation effects using Duan- 
Rach Approach. Siddiq et al. [27] studied the hydromagnetic Radiative stagnation point flow of micropolar nanofluid passed through a shrinking sheet using RKF 45 technique. Awais et al. [28] reported the MHD flow of nanofluid past a stretching surface in the presence of convective cooling which occurs at the boundary has a major role in energy augmentation.

Heat transfer external to curved bodies is also of some significance in biochemical and plastics fabrication processes. Geometrical configurations investigations include circular disks, needles, spheroids, elliptical bodies, cones, truncated cones (frustum) and blunt nosed bodies. Theoretical studies on laminar free convection flow on axisymmetric bodies have received more attention, whether with uniform surface temperature i.e. isothermal conditions (as considered in the present study) or in the case of non-uniform surface temperature and surface heat flux distributions. Hossain and Paul [29] studied the free convection from a vertical permeable circular cone with non-uniform surface temperature. Kairi and Murthy [30] analyzed the effect of viscous dissipation on natural convection heat and mass transfer from vertical cone in a nonNewtonian fluid saturated non-Darcy porous medium. Nadeem and Saleem [31] reported the unsteady mixed convection analytical study of rotating second grade Nanofluid on a rotating cone using similarity transformations and solved analytically using homotopy analysis method. Noghrehabadi et al. [32] investigated the natural convection flow of Nanofluids over a vertical cone embedded in non-Darcy porous media. Nadeem [33] examined the analytical study of third grade fluid over a rotating vertical cone in the presence of nanoparticles. Saleem and Nadeem [34] presented the viscous dissipation and slip effects on a rotating vertical cone in a viscous fluid using homotopy analysis method. Saleem et al. [35] explored the convectional flow of Jeffreys fluid past a rotating cone. Saleem et al. [36] investigated the effects of chemical reaction and heat generation or absorption effects of time-dependent second-order viscoelastic fluid on a rotating cone. All these investigations revealed that heat and flow features are considerably influenced by curvature of the body and more sophisticated thermo fluid behavior is observed than in conventional flat plate (wall) systems.

The objective of the current study is to examine the steady-state, laminar, thermal convection boundary layer flows of third grade non-Newtonian fluid from an isothermal inverted cone. Appropriate non-similar transformations are deployed to render the conservation equations into dimensionless form. The emerging non-dimensional partial differential equations with associated boundary conditions constitute a highly nonlinear, coupled two-point boundary value problem making exact solutions practically impossible. Keller's implicit finite difference "box" scheme is therefore implemented to obtain approximate computational solutions. Validation with 
earlier Newtonian solutions is also documented. The boundary value problem features a number of dimensionless thermophysical parameters, namely the third grade fluid parameter $(\varphi)$, viscoelastic material fluid parameters $\left(\varepsilon_{1}, \varepsilon_{2}\right)$, radiation parameter $(F)$, Prandtl number $(P r)$, heat absorption/generation parameter ( $\Delta)$, magnetic parameter $(M)$ and Buoyancy parameter $(N)$. The influence of various parameters on velocity, temperature, concentration, skin friction number (surface shear stress function), heat transfer rate (local Nusselt number) and mass transfer rate (local Sherwood number) characteristics are studied. The present problem has to the authors' knowledge not appeared thus far in the scientific literature and is relevant to thermal fabrication (heat treatment) of paint sprays, water-based rheological gel solvents and low density polymeric manufacturing processes in chemical engineering.

\section{NON-NEWTONIAN CONSTITUTIVE THIRD GRADE FLUID MODEL}

In the present study we utilize (in part) the rheological properties of a subclass of non-Newtonian fluids known as the third grade fluid. This model physically captures accurately the viscoelastic characteristics of certain polymers [37, 38]. The Cauchy stress tensor of an incompressible third grade non-Newtonian fluid following Truesdell and Noll [39] takes the form:

$$
\tau=-p I+\mu A_{1}+\alpha_{1} A_{2}+\alpha_{2} A_{1}^{2}+\beta_{1} A_{3}+\beta_{2}\left(A_{1} A_{2}+A_{2} A_{1}\right)+\beta_{3}\left(\operatorname{tr} A_{1}^{2}\right) A_{1}
$$

where $\tau$ is the extra stress tensor, $p$ is the pressure, $\boldsymbol{I}$ is the identity tensor, $\alpha_{i}(i=1,2), \beta_{i}(i=1$, 2,3) are the material constants and $A_{k}(k=1,2,3)$ are the first Rivlin-Ericksen tensors [40] which are defined as follows:

$$
\begin{aligned}
& \mathrm{A}_{1}=(\nabla \mathrm{V})+(\nabla \mathrm{V})^{\mathrm{T}} \\
& \mathrm{A}_{n}=\frac{d A_{n-1}}{d t}+A_{n-1}(\nabla \mathrm{V})+A_{n-1}(\nabla \mathrm{V})^{\mathrm{T}} ; n>1
\end{aligned}
$$

The resulting boundary value problem is found to be well-posed and permits a sound methodology for analyzing and appraising non-Newtonian effects on the thermo-fluid polymeric transport phenomena via the deployment of suitable dimensionless parameters.

\section{MATHEMATICAL MODEL}

Steady-state, laminar, double-diffusive, incompressible flow, thermal convection and mass transfer of third grade viscoelastic fluid from an inverted permeable cone with vertex angle $2 A$, is considered, as illustrated in Fig. 1. The vertex of the cone is located at the origin of the coordinate system. The $x$-coordinate is taken along the surface of the cone and $y$-coordinate is 
directed normal to the surface of the cone. The acceleration due to gravity $g$, acts downwards. We also assume that the Boussinesq approximation holds, i.e., the density variation is experienced solely in the buoyancy term in the momentum equation. Both cone and fluid are initially maintained at the same temperature and concentration and are instantaneously raised to a temperature $T_{w}>T_{\infty}$ and concentration $C_{w}>C_{\infty}$, the ambient temperature and concentration of the fluid which remains unchanged. In line with the approach of Sahoo [13] and Hayat [15-17], introducing the boundary layer approximations, the equations for continuity, momentum, energy and spices can be written as follows:

$$
\begin{aligned}
& \frac{\partial(r u)}{\partial x}+\frac{\partial(r v)}{\partial y}=0 \\
& u \frac{\partial u}{\partial x}+v \frac{\partial u}{\partial y}=v \frac{\partial^{2} u}{\partial y^{2}}+\frac{\alpha_{1}}{\rho}\left[u \frac{\partial^{3} u}{\partial x \partial y^{2}}+v \frac{\partial^{3} u}{\partial y^{3}}+\frac{\partial u}{\partial x} \frac{\partial^{2} u}{\partial y^{2}}\right]+\frac{1}{\rho}\left[3 \alpha_{1}+2 \alpha_{2}\right] \frac{\partial u}{\partial y} \frac{\partial^{2} u}{\partial x \partial y} \\
& +\frac{6 \beta_{3}}{\rho}\left(\frac{\partial u}{\partial y}\right)^{2} \frac{\partial^{2} u}{\partial y^{2}}+g \beta\left(T-T_{\infty}\right) \cos A+g \beta^{*}\left(C-C_{\infty}\right) \cos A-\frac{\sigma B_{0}^{2}}{\rho} u \\
& u \frac{\partial T}{\partial x}+v \frac{\partial T}{\partial y}=\alpha \frac{\partial^{2} T}{\partial y^{2}}-\frac{1}{\rho c_{p}} \frac{\partial q_{r}}{\partial y}+\frac{Q_{0}}{\rho c_{p}}\left(T-T_{\infty}\right) \\
& u \frac{\partial C}{\partial x}+v \frac{\partial C}{\partial y}=D_{m} \frac{\partial^{2} C}{\partial y^{2}}
\end{aligned}
$$

The appropriate physical boundary conditions are as follows:

$$
\begin{aligned}
& \text { At } \quad y=0, \quad u=0, \quad v=0, \quad T=T_{w}, \quad C=C_{w} \\
& \text { As } \quad y \rightarrow \infty, \quad u \rightarrow 0, \quad v \rightarrow 0, \quad T \rightarrow T_{\infty}, \quad C \rightarrow C_{\infty}
\end{aligned}
$$

In Eq. (6), the Rosseland diffusion flux model [41, 42] is an algebraic approximation and defined as follows:

$$
q_{r}=\frac{4 \sigma^{*}}{3 k^{*}} \frac{\partial T^{4}}{\partial y}
$$

where $k^{*}$ - mean absorption coefficient and $\sigma^{*}$ - Stefan-Boltzmann constant.

This formulation allows the transformation of the governing integro-differential equation for radiative energy balance into electrostatic potential (Coulomb's law) which is valid for opticallythick media in which radiation only propagates a limited distance prior to experiencing scattering or absorption. It can be shown that the local intensity is caused by radiation emanating from nearby locations in the vicinity of which the emission and scattering are comparable to the location under consideration. For zones where conditions are appreciably different, the radiation has been shown to be greatly attenuated prior to arriving at the location being analyzed. The 
energy transfer depends only on the conditions in the area near the position under consideration. In applying the Rosseland assumption, it is assumed that refractive index of the medium is constant, intensity within the porous medium is nearly isotropic and uniform and wavelength regions exist where the optical thickness is greater than 5 .

Expanding $T^{4}$ using Taylor series and neglecting higher order terms leads to:

$T^{4}=4 T_{\infty}^{3} T-3 T_{\infty}^{4}$

Substituting (10) into (9), the heat conservation equation (6) reduces to:

$u \frac{\partial T}{\partial x}+v \frac{\partial T}{\partial y}=\alpha \frac{\partial^{2} T}{\partial y^{2}}+\frac{16 \sigma^{*} T_{\infty}^{3}}{3 k^{*} \rho c_{p}} \frac{\partial^{2} T}{\partial y^{2}}+\frac{Q_{0}}{\rho c_{p}}\left(T-T_{\infty}\right)$

The stream function, $\psi$, is defined by $r u=\frac{\partial \psi}{\partial y}$ and $r v=-\frac{\partial \psi}{\partial x}$, and the continuity equation is automatically satisfied. Here, $r$, the local radius is defined as, $r(x)=x \sin A$. In order to render the governing equations and the boundary conditions in dimensionless form, the following nondimensional quantities are introduced:

$$
\begin{aligned}
& \xi=\frac{V_{w} x}{v} G r_{x}^{-1 / 4}, \quad \eta=\frac{y}{x} G r_{x}^{1 / 4}, \quad \psi=r v \sqrt[4]{G r_{x}}\left(f+\frac{1}{2} \xi\right), \quad \theta(\xi, \eta)=\frac{T-T_{\infty}}{T_{w}-T_{\infty}}, \quad \operatorname{Pr}=\frac{v}{\alpha} \\
& G r_{x}=\frac{g \beta\left(T_{w}-T_{\infty}\right) x^{3} \cos A}{4 v^{2}}, \quad \varphi=\frac{\beta_{3} v}{\rho x^{4}} G r_{x}^{3 / 2}, \quad \varepsilon_{1}=\frac{\alpha_{1}}{\rho x^{2}} G r_{x}^{1 / 2}, \quad \varepsilon_{2}=\frac{\alpha_{2}}{\rho x^{2}} G r_{x}^{1 / 2}, \quad \phi(\xi, \eta)=\frac{C-C_{\infty}}{C_{w}-C_{\infty}}
\end{aligned}
$$

In view of Eq. (12), the boundary layer Eqs. (5) - (7) reduce to the following coupled, parabolic, nonlinear, dimensionless partial differential equations for momentum, energy and mass for the regime:

$$
\begin{aligned}
& f^{\prime \prime \prime}+\frac{7}{4} f f^{\prime \prime}-\frac{1}{2}\left(f^{\prime}\right)^{2}+\xi f^{\prime \prime}+\varepsilon_{1}\left[\frac{1}{2} f^{\prime} f^{\prime \prime \prime}-\frac{7}{4} f f^{i v}-\xi f^{i v}\right]+\left(3 \varepsilon_{1}+2 \varepsilon_{2}\right) \frac{1}{4}\left(f^{\prime \prime}\right)^{2} \\
& -\left(4 \varepsilon_{1}+2 \varepsilon_{2}\right) \frac{\eta}{4} f^{\prime \prime} f^{\prime \prime \prime}+6 \phi\left(f^{\prime \prime}\right)^{2} f^{\prime \prime \prime}+\theta+N \phi-M f^{\prime} \\
& =\frac{\xi}{4}\left[f^{\prime} \frac{\partial f^{\prime}}{\partial \xi}-f^{\prime \prime} \frac{\partial f}{\partial \xi}-\varepsilon_{1}\left(f^{\prime} \frac{\partial f^{\prime \prime \prime}}{\partial \xi}+f^{\prime \prime \prime} \frac{\partial f^{\prime}}{\partial \xi}-f^{i v} \frac{\partial f}{\partial \xi}\right)-\left(3 \varepsilon_{1}+2 \varepsilon_{2}\right) f^{\prime \prime} \frac{\partial f^{\prime \prime}}{\partial \xi}\right] \\
& \frac{\theta^{\prime \prime}}{\operatorname{Pr}}\left(1+\frac{4}{3 F}\right)+\frac{7}{4} f \theta^{\prime}+\xi \theta^{\prime}+\Delta \theta=\frac{\xi}{4}\left(f^{\prime} \frac{\partial \theta}{\partial \xi}-\theta^{\prime} \frac{\partial f}{\partial \xi}\right) \\
& \frac{\phi^{\prime \prime}}{S c}+\frac{7}{4} f \theta^{\prime}+\xi \theta^{\prime}=\frac{\xi}{4}\left(f^{\prime} \frac{\partial \phi}{\partial \xi}-\phi^{\prime} \frac{\partial f}{\partial \xi}\right)
\end{aligned}
$$

The corresponding transformed boundary conditions are: 
At $\quad \eta=0, \quad f=0, \quad f^{\prime}=0, \quad \theta=1, \quad \phi=1$

As $\quad \eta \rightarrow \infty, \quad f^{\prime} \rightarrow 0, \quad f^{\prime \prime} \rightarrow 0, \quad \theta \rightarrow 0, \quad \phi \rightarrow 0$

Here primes denotes the ordinary differentiation with respect to $\eta, N=\frac{\beta^{*}\left(C_{w}-C_{\infty}\right)}{\beta\left(T_{w}-T_{\infty}\right)}$, $F=\frac{K k^{*}}{4 \sigma^{*} T_{\infty}^{3}}$ and $\Delta=\frac{Q_{0} x^{2}}{\rho v c_{p} \sqrt{G r_{x}}}$. The skin-friction coefficient (shear stress at the cone surface), heat transfer rate (local Nusselt number) and mass transfer rate (local Sherwood number) at the cone surface are defined as follows:

$$
\begin{aligned}
& G r^{-3 / 4} C_{f}=f^{\prime \prime}(\xi, 0)+\varepsilon_{1}\left(\frac{5}{4} f^{\prime} f^{\prime \prime}(\xi, 0)-\frac{7}{4} f f^{\prime \prime}(\xi, 0)\right)+2 \varphi\left(f^{\prime \prime}(\xi, 0)\right)^{3} \\
& G r^{-1 / 4} N u=-\theta^{\prime}(\xi, 0) \\
& S h_{x} G r_{x}^{1 / 4}=-\phi^{\prime}(\xi, 0)
\end{aligned}
$$

In vicinity of the lower stagnation point, $\xi \sim 0$ and the boundary layer equations (13) - (15) reduce to a system of ordinary differential equations:

$$
\begin{aligned}
f^{\prime \prime \prime}+\frac{7}{4} f f^{\prime \prime}-\frac{1}{2}\left(f^{\prime}\right)^{2}+ & \varepsilon_{1}\left[\frac{1}{2} f^{\prime} f^{\prime \prime \prime}-\frac{7}{4} f f^{i v}\right]+\left(3 \varepsilon_{1}+2 \varepsilon_{2}\right) \frac{1}{4}\left(f^{\prime \prime}\right)^{2} \\
& -\left(4 \varepsilon_{1}+2 \varepsilon_{2}\right) \frac{\eta}{4} f^{\prime \prime} f^{\prime \prime \prime}+6 \phi\left(f^{\prime \prime}\right)^{2} f^{\prime \prime \prime}+\theta+N \phi-M f^{\prime}=0
\end{aligned}
$$

$\frac{\phi^{\prime \prime}}{S c}+\frac{7}{4} f \phi^{\prime}=0$

The general model is solved using a powerful and unconditionally stable finite difference technique introduced by Keller [43]. The Keller-box method has a second order accuracy with arbitrary spacing and attractive extrapolation features. It converges quickly and is ideal for parabolic problems.

\section{COMPUTATIONAL SOLUTION}

An implicit difference Keller-Box method is implemented to solve the non-linear boundary layer Eqs. (13) - (15) subject to the boundary conditions (16). This technique has remained extremely popular and maintained comparably efficient than other numerical methods such as finite element, boundary elements, spectral methods etc. Keller-Box method has a second order accuracy with arbitrary spacing and attractive extrapolation features. It is unconditionally stable 
and achieves exceptional accuracy. It converges quickly and provides stable numerical meshing features and provides an improvement in accuracy on explicit or semi-implicit schemes and utilizes customizable stepping in a fully implicit approach. Relevant details are provided in Keller [43]. The Keller-Box discretization is fully coupled at each step which reflects the physics of parabolic systems - which are also fully coupled. Discrete calculus associated with the Keller-Box scheme has also been shown to be fundamentally different from all other mimetic (physics capturing) numerical methods, as elaborated in Abdul gaffar et al. [44 - 48].

\section{NUMERICAL RESULTS AND DISCUSSION}

A comprehensive set of numerical results have been obtained and are illustrated in Figs. 2 - 11 and tables 1 - 3. The numerical problem comprises of two independent variables $(\xi, \eta)$, three dependent fluid dynamic variables $(f, \theta, \phi)$ and six rheological and thermo-physical parameters, viz., $\varphi, \varepsilon_{1}, \varepsilon_{2}, F, M, N, \Delta$, Sc, $\operatorname{Pr}$. The following default parameter values are deployed: $\varphi=0.1$, $\varepsilon_{1}=\varepsilon_{2}=0.3, F=0.5, M=0.5, N=0.5, \operatorname{Pr}=7.0, \Delta=0.1, S c=0.6$ and $\xi=1.0$. Furthermore, the influence of stream-wise coordinate on flow, temperature and concentration characteristics is also investigated. The selection of data is consistent with established works in the field; specifically for third grade fluids we have adopted data from Sahoo and Poncet [11] which is in turn consistent with Truesdell and Noll [39]. The present model reduces to the Newtonian isothermal solid cone version of the Hossain-Paul [29] model when non-isothermal wall index and wall suction are set to zero in their general model and when $\operatorname{Pr}=0.1$ (low density polymer), $\varepsilon_{1}=\varepsilon_{2}=\varphi=0$ (third grade viscoelastic effects vanish). The comparison solutions are documented in Table 1 and demonstrate excellent correlation for the heat transfer rate, $-\theta^{\prime}(\xi, 0)$ for various values of $\xi$. With increasing tangential coordinate there is evidently a strong enhancement in heat transfer rates. Table 2 provides KBM solutions for the influence of the magnetic parameter, $M$ and the buoyancy parameter, $N$, on skin friction, heat transfer rate and mass transfer rate along with the variation in $\xi$. In Table 2, we observe that with increasing $M$ values, the skin friction is reduced. Also a slight decrease is observed in heat transfer rate and mass transfer rate. Increasing $N$ is observed to increase skin friction, heat transfer rate and mass transfer rate. In Table 3, we found that with increasing $F$ values, the skin friction and mass transfer rate are reduced, whereas, the heat transfer rate is enhanced. And an increasing $\Delta$ is observed to decrease skin friction and heat transfer rate but the mass transfer rate is slightly increased. 
Figures 2(a) - 2(c) depicts the velocity $\left(f^{\prime}\right)$, temperature $(\theta)$ and concentration $(\phi)$ distributions with increasing third grade material fluid parameter $(\varphi)$ through the boundary layer regime. There is a strong elevation (Fig. 2(a)) in linear velocity closer to the cone surface with an increase in $\varphi$. Hence, the momentum boundary layer thickness is decreased with greater third order viscoelastic parameter. The mathematical model reduces to the Newtonian viscous flow model as $\varphi \rightarrow 0, \varepsilon_{1} \rightarrow 0$ and $\varepsilon_{2} \rightarrow 0$. The momentum boundary layer equation in this case contracts to the familiar equation for Newtonian convection from a cone, viz:

$$
f^{\prime \prime \prime}+\frac{7}{4} f f^{\prime \prime}-\frac{1}{2}\left(f^{\prime}\right)^{2}+\xi f^{\prime \prime}+\theta+N \phi+M f^{\prime}=\frac{\xi}{4}\left[f^{\prime} \frac{\partial f^{\prime}}{\partial \xi}-f^{\prime \prime} \frac{\partial f}{\partial \xi}\right]
$$

Greater third order material effects therefore serve to marginally thicken thermal boundary layers. The third grade material parameter, $\varphi$, is given by $\frac{\beta_{3} v}{\rho x^{4}} G r_{x}^{3 / 2}$ where $G r_{x}=\frac{g \beta\left(T_{w}-T_{\infty}\right) x^{3} \cos A}{4 v^{2}}$ is the local thermal Grashof number. From careful inspection of the parameter, $\varphi$, it emerges that $\varphi$ is directly proportional to third grade material parameter $\left(\beta_{3}\right)$ and inversely proportional to the square of kinematic viscosity $\left(v^{2}\right)$. This results in acceleration in the boundary layer flow i.e. greater $f^{\prime}$ values as observed in fig. 2 a. The $\varphi$ parameter actually arises in a single term in only the linear momentum equation (13), viz $+6 \varphi\left(f^{\prime \prime}\right)^{2} f^{\prime \prime \prime}$, and is therefore strongly related to shear rate. As $\varphi$ is increased, the fluid requires a lesser shear to flow and stronger elastic effects are present which encourage flow acceleration. The effect is most prominent near the cone surface and is reversed further towards the freestream. However, the acceleration effect in the near-wall region is substantially greater than the retardation effect at the edge of the boundary layer i.e. the latter is a weaker phenomenon. The temperature field (Eq. 14) is indirectly influenced by the parameter $\varphi$ again owing to coupling with linear momentum Eq. (13) via the thermal buoyancy term $(\theta)$. There is a slight increase in temperature magnitudes in fig. 2(b) with a rise in $\varphi$. The thermal boundary layer thickness is therefore enhanced with greater rheological effect. The decrease in viscosity associated with greater $\varphi$ values implies that momentum diffusion rate is lower relative to thermal diffusion rate in the boundary layer. This results in elevated heat diffusion which causes temperatures to increase, a trend which is sustained across the boundary layer regime. In fig. 2(c) with increasing $\varphi$ there is a slight increase in concentration $(\phi)$. We emphasize that the selection of parameters associated with figs. 2(a) - (c) (and indeed all other subsequent graphical plots), is deliberate. Unfortunately the vast 
majority of studies using the third grade model do not elaborate on the physical reasons for selection of material parameter values. They arbitrarily specify such parameters and this makes it difficult to apply the solutions to real engineering polymeric flows. While such studies are mathematically rigorous they are often exercises in analysis and not in physical interpretationsee [13-20]. In the constitutive Eq. (1), Truesdell and Noll [39] have shown that for proper description of third grade fluids, if all the motions of such liquids are to be compatible with thermodynamics in the sense that these motions meet the Clausius-Duhem inequality and if it is assumed that the specific Helmholtz free energy is minimum when the fluid is locally at rest, then the following conditions must hold:

$\mu \geq 0, \alpha_{1} \geq 0,\left|\alpha_{1}+\alpha_{2}\right| \leq \sqrt{24 \mu \beta_{3}}, \beta_{1}=\beta_{2}=0, \beta_{3} \geq 0$.

The specification of $\varepsilon_{1}=\varepsilon_{2}=0.3$ as defined in Eq. (13) relates to the prescription of the material moduli values $\alpha_{1}, \alpha_{2}$ in the Reiner-Rivlin third grade viscoelastic model i.e. Eq. (24). Evidently, the third grade material parameter $\left(\beta_{3}\right)$ can have values greater or equal to zero, resulting in $\varphi$ values dependent on the particular selection. Based on consistency with the work of Akyildiz et al. [49] and Bég et al. [50], we study weakly elastic fluids as characteristic of solvents and specify $\varepsilon_{l}=\varepsilon_{2}=0.3$. All computations correspond to a $\xi=1.0$ i.e. some distance downstream from the leading edge $(\xi=0.0)$ on the curved surface of the cone. The solutions given are at a general location and not confined to extremities of the cone geometry.

Figures 3(a) - 3(c) illustrates the effect of the first material viscoelastic fluid parameter, $\varepsilon_{1}$, on the velocity $\left(f^{\prime}\right)$, temperature $(\theta)$ and concentration $(\phi)$. The parameter, $\varepsilon_{l}$, is directly proportional to first material viscoelastic modulus, $\alpha_{1}$. It appears in numerous terms in the linear momentum Eq. (13). As $\varepsilon_{l}$ increases, the linear velocity decrease (fig. 3a). This is probably due to the relaxation in the rheological fluid with further separation from the cone surface. This results in a shear-thickening in the fluid and higher viscosity which slows the boundary layer flow in this region leading to an increase in momentum boundary layer thickness. Fig. $3 \mathrm{~b}$ shows that temperatures are consistently enhanced throughout the boundary layer regime with greater $\varepsilon_{1}$ values. The reduction in liquid viscosity results in energy diffusion rate exceeding the momentum diffusion rate which heats the boundary layer and increases thermal boundary layer thickness. Concentration is found in fig. $3 \mathrm{c}$ to be markedly increased with greater values of first material viscoelastic fluid parameter, $\varepsilon_{1}$.

Figures 4(a) - 4(c) displays the evolution of velocity $\left(f^{\prime}\right)$, temperature $(\theta)$ and concentration $(\phi)$ functions with a variation in the second material fluid parameter $\varepsilon_{2}$. Dimensionless velocity 
component is observed to be substantially enhanced with increasing $\varepsilon_{2}$ values. The definitions of $\varepsilon_{1}$ and $\varepsilon_{2}$ only differ in the material modulus $\left(\alpha_{1}\right.$ and $\left.\alpha_{2}\right)$ included. However the influence on thermo-fluid characteristics is very different. Acceleration is consistently achieved with greater $\varepsilon_{2}$ values, at any location in the boundary layer transverse to the cone surface (fig. 4a), in contrast to increasing $\varepsilon_{l}$ (fig. 3a) where a different response is induced depending on the location in the boundary layer. Larger $\varepsilon_{2}$ values correspond to an effective reduction in the viscosity of the liquid and greater elasticity. Contrary to fig. $3 \mathrm{~b}$, where temperatures are elevated with higher $\varepsilon_{1}$ values, in fig. $4 \mathrm{~b}$ we observe that temperatures are reduced with larger $\varepsilon_{2}$ values. Heat diffusion rate is therefore lower with higher $\varepsilon_{2}$ values indicating that thermal boundary layer thickness is lowered. Concentration is found in fig. $4 \mathrm{c}$ to be consistently reduced with higher magnitudes of second material viscoelastic fluid parameter, $\varepsilon_{2}$.

Figures 5(a) - 5(c) presents the influence of the radiation parameter $(F)$ on the velocity $\left(f^{\prime}\right)$, temperature $(\theta)$ and concentration $(\phi)$ distributions. We observe in Fig. 5(a) that an increase in $F$, strongly decelerates the flow i.e., depresses linear velocity; this trend is sustained until a certain distance normal to the cone surface after which a transition occurs. This parameter appears in the energy conservation Eq. (14). $F=\frac{K k *}{4 \sigma^{*} T_{\infty}^{3}}$ represents the thermal conduction to the thermal radiation heat transfer. Therefore, temperature is decreased with increasing values of $F$, as observed in fig. 5(b). With increasing $F$ values there is also a progressive enhancement in concentration as seen in fig. 5(c).

Figures 6(a) - 6(c) depict the velocity $\left(f^{\prime}\right)$, temperature $(\theta)$ and concentration $(\phi)$ distributions for various values of heat generation or absorption parameter, $\Delta$. With increasing values of heat generation $(\Delta>0)$ the velocity and temperature are significantly accelerated but the concentration is slightly decelerated. Whereas, with heat absorption $(\Delta<0)$ the flow is retarded, thermal boundary layer thickness is reduced whereas the concentration boundary layer thickness is increased.

Figures 7(a) - 7(c) illustrates the effects of velocity $\left(f^{\prime}\right)$, temperature $(\theta)$ and concentration $(\phi)$ distributions for various values of the magnetic parameter, $M$. It is seen that with increasing values of $M$, the flow decelerates i.e., velocity decreases. However, with increasing $M$, the temperature and concentration are enhanced. The Hartmann number, $M$, simulates the relative contribution of Lorentzian magnetohydrodynamics drag force relative to viscous hydrodynamic force. As $M$ increases, greater opposition is generated to the flow past the cone leading to 
deceleration. The supplementary work expended in dragging the polymer against the imposition of the transverse magnetic field creates heating the polymer. This dissipation of heat leads to rise in temperature and thickening of thermal boundary layers in polymers. This phenomenon is extensively presented in magnetohydrodynamics studies $[52,53]$.

Figures 8(a) - 8(c) presents the profiles for velocity $\left(f^{\prime}\right)$, temperature $(\theta)$ and concentration $(\phi)$ distributions for various values of the buoyancy ratio parameter, $N$. For $N>0$, the flow is accelerated. Initially for $N<0$ i.e. the buoyancy opposed case where thermal and species buoyancy forces act against each other, the flow is decelerated. Further, from cone surface there is a transition in the influence of $N . N>0$ leads to a slight reduction in velocity with the contrary for $N<0$. The influence of a large change in $N$ is much less pronounced further from the wall. Buoyance forces therefore exert a much more marked effect in the vicinity of the cone surface. A very response is sustained by temperature and concentration for different values of $N$. The parameter $N=\frac{\beta^{*}\left(C-C_{\infty}\right)}{\beta\left(T-T_{\infty}\right)}$ expresses the concentration to thermal buoyancy force ratio. For cases where $N<1$, thermal buoyancy will dominate concentration buoyancy effects and vice versa for $N>1$.

Figures 9(a) - 9(c) depict the velocity $\left(f^{\prime}\right)$, temperature $(\theta)$ and concentration $(\phi)$ distributions with radial coordinate, for various stream-wise coordinate values, $\xi$. This parameter also manifest the local Grashof number and can be viewed as a free convection parameter as elaborated by Gorla et al. [53]. Clearly, from fig. 9(a) it is observed that as $\xi$ increases, the fluid velocity decreases. This is due to the fact that with greater streamwise coordinate, the flow location moves along the cone surface from the apex towards the broad periphery of the cone. Buoyancy forces increase as this occurs and these suppress momentum diffusion, leading to deceleration in the flow and a thicker boundary layer structure. All the temperature and concentration profiles (fig. 9(b) \& 9(c) respectively) decay smoothly from the maximum at the cone surface to the minimum in the free stream. With progressive distance from the leading edge (cone apex), the fluid is therefore cooled and thermal boundary layer thickness decreases.

Figures 10(a) - 10(c) depict the influence of the third grade dimensionless material parameter, $\varphi$, on the dimensionless skin friction coefficient $\left(C_{f}\right)$, heat transfer rate i.e. Nusselt number $\left(N_{u}\right)$ and mass transfer rate i.e. Sherwood number $(S h)$ at the cone surface. In fig. 10(a) It is observed that the $C_{f}$ is enhanced with an increase in $\phi$. Since higher skin friction corresponds to greater acceleration and larger values of third grade material parameter are known to reduce viscosity 
effects and enhance momentum diffusion, whereas, the surface heat transfer rate (fig. (10b)) is reduced substantially with increasing $\varphi$ which again correlates well with temperature computations discussed previously. Since temperatures decrease with greater third grade material viscoelastic effect, heat transfer to the wall must also fall (heat transfer is enhanced to the body of fluid) and this explains why Nusselt number magnitudes are reduced. Fig. 10(c) shows that mass transfer rate $(S h)$ is considerably reduced with greater $\phi$.

Figures 11(a) - 11(c) illustrate the effect of the material fluid parameter $\varepsilon_{l}$ on the dimensionless skin friction $\left(C_{f}\right)$, heat transfer rate i.e. Nusselt number $\left(N_{u}\right)$ and mass transfer rate i.e. Sherwood number $(S h)$ at the cone surface. It is observed that the $C_{f}$ and $N_{u}$ are depressed strongly along the entire cone surface i.e. for all values of $\xi$, with an increase in $\varepsilon_{l}$, conversely, $S h$ is significantly elevated with increasing $\varepsilon_{l}$. The first viscoelastic material modulus parameter decelerates the linear flow whereas it raises temperatures (see figs 3(a)-3(c)). This is entirely consistent with the results given in figs. 11(a) - 11(c) wherein skin friction and wall heat transfer rate (Nusselt number) are depressed whereas the mass transfer rate is elevated.

\section{CONCLUSIONS}

Numerical results are presented for the buoyancy-driven, non-similar, boundary layer flow of third grade viscoelastic non-Newtonian fluid external to an isothermal vertical cone. The Kellerbox implicit second order accurate finite difference numerical scheme has been utilized to efficiently solve the transformed, dimensionless velocity and thermal boundary layer equations, with prescribed boundary conditions. A comprehensive assessment of the effects of the third grade parameter $(\varphi)$, first and second viscoelastic material fluid parameters $\left(\varepsilon_{1}, \varepsilon_{2}\right)$, thermal radiation parameter $(F)$, heat generation/absorption parameter $(\Delta)$, Prandtl number $(\operatorname{Pr})$, magnetic parameter $(M)$ and Buoyancy ratio parameter $(N)$ and also the streamwise coordinate ( $\xi$ ) on thermo-fluid characteristics has been conducted. Very stable and accurate solutions are obtained with the present finite differences code. Validation of the implicit Keller box method (KBM) solutions has been achieved with earlier Newtonian solutions. The computations have shown that the different third grade rheological parameters exert a varied influence on velocity, temperature and concentration functions, and also on the gradients of these functions (i.e. skin friction, Nusselt number and Sherwood number). Heat transfer rate and mass transfer rate are markedly reduced and skin friction is enhanced for all values of $\varphi$. With greater values of first viscoelastic material parameter $\left(\varepsilon_{1}\right)$ skin friction and heat transfer rate are significantly reduced whereas the mass transfer is enhanced. Increasing third grade material parameter $(\phi)$ is seen to 
decrease linear velocity and slightly increases temperature and concentration magnitudes. Increasing stream-wise coordinate $(\xi)$ decelerates the boundary layer flow and cools the boundary layer. The Keller-box code is able to solve nonlinear rheological boundary layer flow problems very efficiently and therefore presents excellent promise in simulating transport phenomena in other non-Newtonian fluids. In this regard it is being explored with other nonNewtonian formulations and the results of these studies will be communicated imminently.

\section{REFERENCES}

[1] T. F. Irvine, J. Karni, Non-Newtonian fluid flow and heat transfer, Handbook of Single-Phase Convective heat transfer, Chapter 20, Wiley New York, 20.1 - 20.57, 1987.

[2] A. SubbaRao, V.R. Prasad, K. Harshavalli, O. Anwar Bég, Thermal radiation effects on nonNewtonian fluid in a variable porosity regime with partial slip, J. Porous Media, Vol. 19, Issue 4, pp.313-329, 2016.

[3] Y. Y. Lok, I. Pop, D. B. Ingham, Oblique stagnation slip flow of a micropolar fluid, Meccanica, Vol. 45, Issue 2, pp.187-198, 2010.

[4] Azad Hussain, Anwar Ullah, Boundary layer flow of a Walter's B fluid due to a stretching cylinder with temperature dependent viscosity, Alexandria Engineering Journal, Vol. 55, Issue 4, pp.3073-3080, 2016.

[5] S. Abdul Gaffar, V. Ramachandra Prasad, E. Keshava Reddy, Computational study of Jeffrey's non-Newtonian fluid past a semi-infinite vertical plate with thermal radiation and heat generation/absorption, Ain Shams Engineering Journal, Vol. 8, Issue 2, pp.277-294, 2017.

[6] A. Subba Rao, C.H. Amanulla, N. Nagendra, O. Anwar Beg, A. Kadir, Hydromagnetic flow and heat transfer in a Williamson Non-Newtonian fluid from a Horizontal circular cylinder with Newtonian Heating, Int. J. Applied and Computational Mathematics, 2017. DOI: $10.1007 / \mathrm{s} 40819-017-0304-\mathrm{x}$

[7] Ramachandra Prasad V., S. Abdul gaffar and O. Anwar Bég, Non-Similar computational solutions for free convection boundary layer flow of a nanofluid from an isothermal sphere in a Non-Darcy porous medium, J. Nanofluids, Vol. 4, No. 2, pp.203-213, 2015.

[8] Zulfiqar Ali Zaidi, Syed Tauseff Mohyud-Din, Effect of joule heating and MHD in the presence of convective boundary condition for upper convected Maxwell fluid through wall jet, J. Molecular Liquids, Vol. 230, pp.230-234, 2017.

[9] S. Abdul Gaffar, V. Ramachandra Prasad, O. Anwar Beg, Computational study of nonNewtonian Eyring-Powell fluid from a vertical porous plate with Biot number effects, $J$. of the 
Brazilian Society of Mechanical Sciences and Engineering, Vol. 39, Issue 7, pp.2747-2765, 2017.

[10] S. Abdul Gaffar, V. Ramachandra Prasad, E. Keshava Reddy, Computational Study of MHD free convection flow of non-Newtonian Tangent Hyperbolic fluid from a vertical surface in porous media with Hall/Ionslip current and Ohmic dissipation, International Journal of Applied and Computational Mathematics, Vol. 3, Issue 2, pp.859-890, 2017.

[11] Rashid Mehmood, S. Nadeem, S. Saleem, Noreen Sher Akbar, Flow and Heat Transfer analysis of a Jeffery Nano fluid impinging obliquely over a stretched plate, Journal of the Taiwan Institute of Chemical Engineers, Vol. 74 (2017) 49-58.

[12] S. Nadeem, S. Saleem, An optimized study of mixed convection flow of a rotating Jeffrey nanofluid on a rotating vertical cone. Journal of Computational and Theoretical Nanoscience, Vol. 12, No. 10, pp.3028-3035, 2015.

[13] B. Sahoo, S. Poncet, Flow and heat transfer of third grade fluid past an exponentially stretching sheet with partial slip boundary conditions, Int. J. Heat and Mass Transfer, Vol. 54, Issue 23-24, pp.5010-5019, 2011.

[14] A. Aziz and T. Aziz, MHD flow of a third grade fluid in porous half space with plate suction or injection: An analytical approach, Applied Mathematics and Computation, Vol. 218, Issue 21, pp.10443-1045, 2012.

[15] T. Hayat, A. Shafiq, A. Alsaedi, M. Awais, MHD axisymmetric flow of third grade fluid between stretching sheets with heat transfer, Computers \& Fluids, Vol. 86, pp.103-108 (2013).

[16] Hayat T., Zahid Iqbal, Meraj Mustafa and Awatif A. Hendi, Melting heat transfer in the stagnation-point flow of third grade fluid past a stretching sheet with viscous dissipation, Thermal Science, Vol. 17, pp.865-875 (2013).

[17] Hayat T., Anum Shafiq, A. Alsaedi, MHD axisymmetric flow of third grade fluid by a stretching cylinder, Alexandria Engineering J., Vol. 54, pp.205-212 (2015).

[18] Samuel O. Adesanya, J.A. Falade, Thermodynamic analysis of hydromagnetic third grade fluid flow through a channel filled with porous medium, Alexandria Engineering J., Vol. 54, pp.615-622, 2015.

[19] Abdul hameed M., W.Varnhorn, I. Hasim, R. Roslan, The unsteady flow of a third grade fluid caused by the periodic motion of an infinite wall with transpiration, Alexandria Engineering J., Vol. 54, Issue 4, pp.1233-1241, 2015. 
[20] Rashidi M.M., S. Bagheri, E. Momoniat, N. Freidoonimehr, Entropy analysis of convective MDH flow of third grade non-Newtonian fluid over a stretching sheet, Ain Shams Engineering J. (2015). http://dx.doi.org/10.1016/j.asej.2015.08.012

[21] Aracely Lopez, Guillermo Ibanez, Joel Pantoja, Joel Moreira, Orlanda Lastres, Entropy generation analysis of MHD nanofluid flow in a porous vertical microchannel with nonlinear thermal radiation, slip flow and convective-radiative boundary conditions, Int. J. Heat and Mass Transfer, Vol. 107, pp.982-994, 2017.

[22] M. Rashad, Impact of thermal radiation on MHD slip flow of a ferrofluid over a nonisothermal wedge, Journal of Magnetism and Magnetic Materials, Vol. 422, pp.25-31, 2017.

[23] T. Hayat, Sajid Qayyum, Sabit Ali Shehzad, Ahmed Alsaedi, Simultaneous effects of heat generation/absorption and thermal radiation in magnetohydrodynamics (MHD) flow of Maxwell nanofluid towards a stretched surface, Results in Physics, 2017. http://dx.doi.org/10.1016/j.rinp.2016.12.009

[24] T. Hayat, Madiha Rashid, Maria Imtiz, Ahmed Alsaedi, MHD convective flow due to a curved surface with thermal radiation and chemical reaction, J. of Molecular Liquids, Vol. 225, pp.482-489, 2017.

[25] Jalilpour, S. Jafarmadar, M.M. Rashidi, D.D. Ganji, R. Rahime, A.B. Shotorban, MHD nonorthogonal stagnation point flow of a nanofluid towards a stretching surface in the presence of thermal radiation, Ain Shams Engineering Journal, 2017. http://dx.doi.org/10.1016/j.asej.2016.09.011

[26] S. Dogonchi, K. Divsalar, D.D. Ganji, Flow and heat transfer of MHD nanofluid between parallel plates in the presence of thermal radiation, Computer Methods in Applied Mechanics and Engineering, Vol. 310, pp.58-76, 2016.

[27] M.K. Siddiq, A. Rauf, S.A. Shehzad, F.M. Abbasi, M.A. Meraj, Thermally and solutally convective radiation in MHD stagnation point flow of micropolar nanofluid over a shrinking sheet, Alexandria Engineering Journal, 2017. http://dx.doi.org/10.1016/j.aej.2017.01.019 [28] M. Awais, S. Saleem, T. Hayat, S. Irum, Hydromagnetic couple-stress nanofluid flow over a moving convective wall: OHAM analysis, Acta Astronautica, Vol. 129, pp.271-276, 2016.

[29] Hossain M.A., S. C. Paul, Free convection from a vertical permeable circular cone with non-uniform surface temperature, Acta Mechanica, Vol. 151, pp.103-114 (2001).

[30] R.R. Kairi, PVSN Murthy, Effect of viscous dissipation on natural convection heat and mass transfer from vertical cone in a non-Newtonian fluid saturated non-Darcy porous medium, Applied Math. Computation, Vol. 217, pp.44-48 (2011) 
[31] S. Nadeem, S. Saleem, 2014, Analytical Study of Rotating Non-Newtonian Nanofluid on a Rotating Cone, Journal of Thermophysics and heat transfer, Vol. 28, No. 2, pp.295-302, 2014

[32] Noghrehabadi A., A. Behseresht, M. Ghalambaz, Natural convection flow of nanofluids over a vertical cone embedded in non-Darcy porous media, AIAA J. Thermophysics and Heat Transfer, Vol. 27, pp.334-341 (2013).

[33] Nadeem S. and S. Saleem, Analytical study of third grade fluid over a rotating vertical cone in the presence of nanoparticles, Int. J. Heat Mass Transfer, Vol. 85, pp.1041-1048 (2015).

[34] S. Saleem, S. Nadeem, Theoretical analysis of slip flow on a rotating cone with viscous dissipation effects, Journal of Hydrodynamics, Ser. B, Vol. 27, Issue 4, pp. 616-623, 2015

[35] S. Saleem, S. Nadeem, N. Sandeep, A Mathematical Analysis of Time Dependent Flow On a Rotating Cone In a Rheological Fluid, Propulsion and Power Research, Vol. 6, Issue 3, pp.233-241, 2017.

[36] S. Saleem, S. Nadeem, Mohammad Awais, Time dependent second order viscoelastic fluid flow on a rotating cone with Heat generation and chemical reaction, Journal of Aerospace Engineering, Vol. 29, Issue 4, 04016009, 2016.

[37] Bird, R.B., Armstrong, R.C. and Hassager, O., Dynamics of Polymeric Liquids. Volume 1: Fluid Mechanics, Vol. 1, 2nd Edition, Wiley Interscience, New York, USA (1987).

[38] Larson, R.G., Constitutive Equations for Polymer Melts and Solutions, Series in Chemical Engineering, Butterworths, Boston (1988).

[39] Truesdell C. and Noll W., The non-linear field theories of mechanics, Handbuch der Physik, Band III/3, pp. 1-602, Springer, Berlin, Germany (1965).

[40] Rivlin R. S. and Ericksen J. L., Stress-deformation relations for isotropic materials, Journal of Rational Mechanics and Analysis, vol. 4, pp. 323-425, 1955.

[41] Gorla, R. S. R. "Radiative effect on conjugate forced convection in a laminar wall jet along a flat plate." Encyclopaedia Fluid Mechanics, Suppl. 3: Advances in Flows Dynamics, Gulf Publishing, Texas, USA (1993).

[42] Bég, O. Anwar, J. Zueco, S. K. Ghosh and A. Heidari. "Unsteady magnetohydrodynamic heat transfer in a semi-infinite porous medium with thermal radiation flux: analytical and numerical study." Advances in Numerical Analysis 2011 (2011): 1-17.

[43] Keller H.B., Numerical methods in boundary-layer theory, Ann. Rev. Fluid Mech. 10, 417433 (1978). 
[44]S. Abdul Gaffar, V. Ramachandra Prasad, E. Keshava Reddy, Non-Newtonian thermal convection from an isothermal sphere with Biot number effects, Int. $J$ of Industrial Mathematics, Vol.8, No.2, 2016

[45]S. Abdul Gaffar, V. Ramachandra Prasad, E. Keshava Reddy, O. Anwar Beg, Magnetohydrodynamic free convection boundary layer flow of non-Newtonian Tangent Hyperbolic fluid from a vertical permeable cone with variable surface temperature, J of Brazilian Society of Mechanical Sciences and Engineering, Vol. 39, Issue 1, pp.101-116, 2017.

[46] S. Abdul Gaffar, V. Ramachandra Prasad E. Keshava Reddy, MHD free convection flow of non-Newtonian Eyring-Powell fluid from vertical surface in porous media with Hall/Ionslip currents and Ohmic dissipation, Alexandria Engineering Journal, Vol.55, pp.875-905, 2016.

[47] S. Abdul Gaffar, V. Ramachandra Prasad, E. Keshava Reddy, Mixed Convection Boundary layer flows of a Non-Newtonian Jeffrey's fluid from a non-isothermal wedge, Ain Shams Engineering Journal, Vol. 8, Issue 2, pp.145-162, 2017

[48] S. Abdul Gaffar, V. Ramachandra Prasad, E. Keshava Reddy, O. Anwar Beg, Magnetohydrodynamic free convection boundary layer flow of non-Newtonian Tangent Hyperbolic fluid from a vertical permeable cone with variable surface temperature, J of Brazilian Society of Mechanical Sciences and Engineering, Vol. 39, Issue 1, pp.101-116, 2017.

[49] T. Akyildiz, F., Bellout, H. and Vajravelu, K.: Exact solutions of nonlinear differential equations arising in third grade fluid flows, Int. J. Non-Linear Mech., 39, 1571-1578 (2004).

[50] O. Anwar Bég, H. S. Takhar, R. Bharagava, Rawat, S. and Prasad, V.R., Numerical study of heat transfer of a third grade viscoelastic fluid in non-Darcian porous media with thermophysical effects, Physica Scripta, 77, 1-11 (2008).

[51] O. D. Makinde, K. Zimba, O. Anwar Bég, Numerical study of chemically-reacting hydromagnetic boundary layer flow with Soret/Dufour effects and a convective surface boundary condition, Int. J. Thermal and Environmental Engineering, 4, 89-98 (2012).

[52] A. J. Chamkha, M. Mujtaba, A. Quadri, and C. Issa, Thermal radiation effects on MHD forced convection flow adjacent to a non-isothermal wedge in the presence of a heat source or sink, Heat and Mass Transfer, 39, 305-312 (2003).

[53] R.S.R. Gorla and S. Nakamura, Combined convection from a rotating cone to micropolar fluids, Math. Modelling Sci.Comput., 2, 1249-1254 (1993). 


\section{FIGURES}

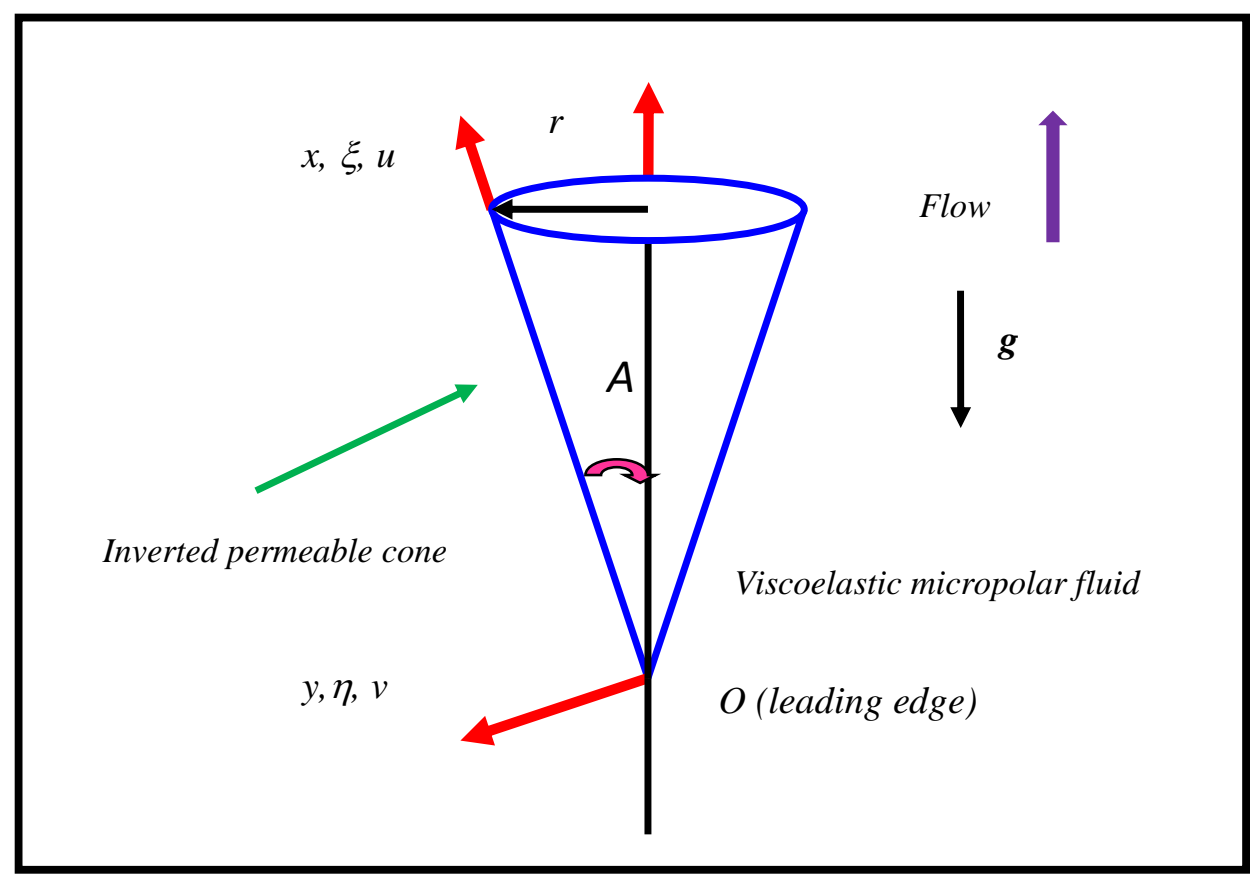

Fig. 1 Physical model and coordinate system

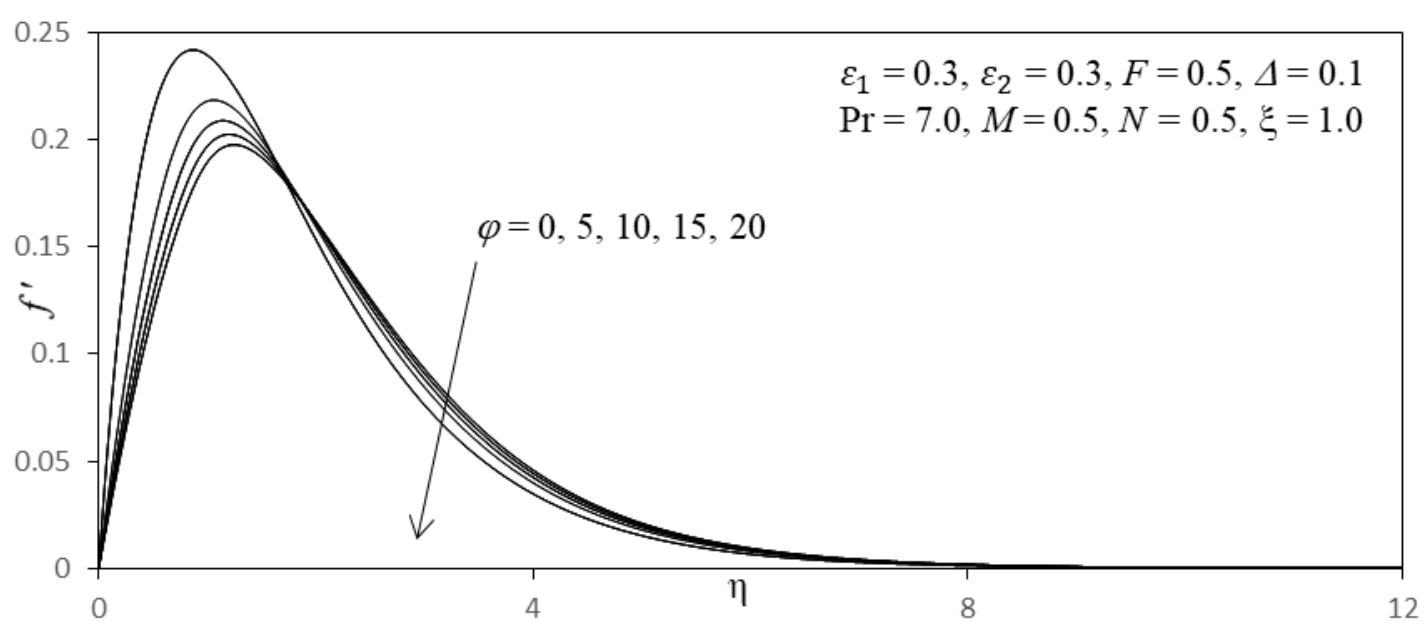

Fig.2(a) Effect of $\varphi$ on Velocity Profiles 


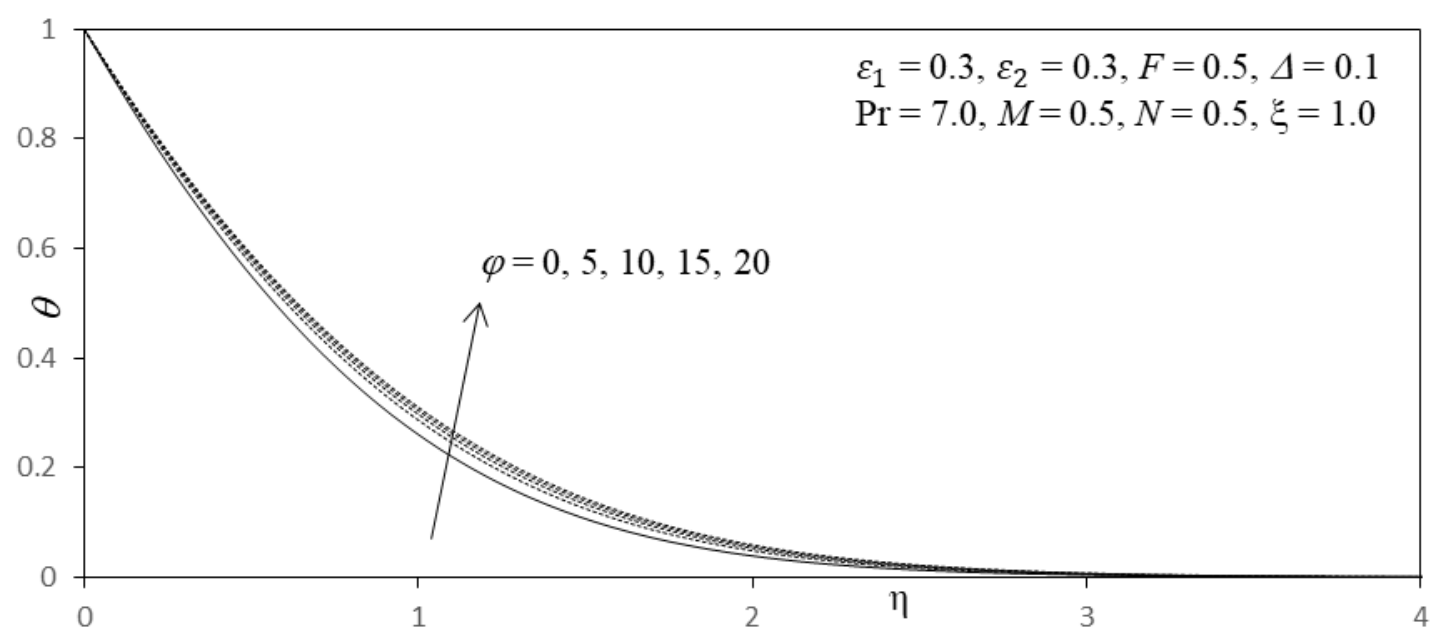

Fig.2(b) Effect of $\varphi$ on Temperature Profiles

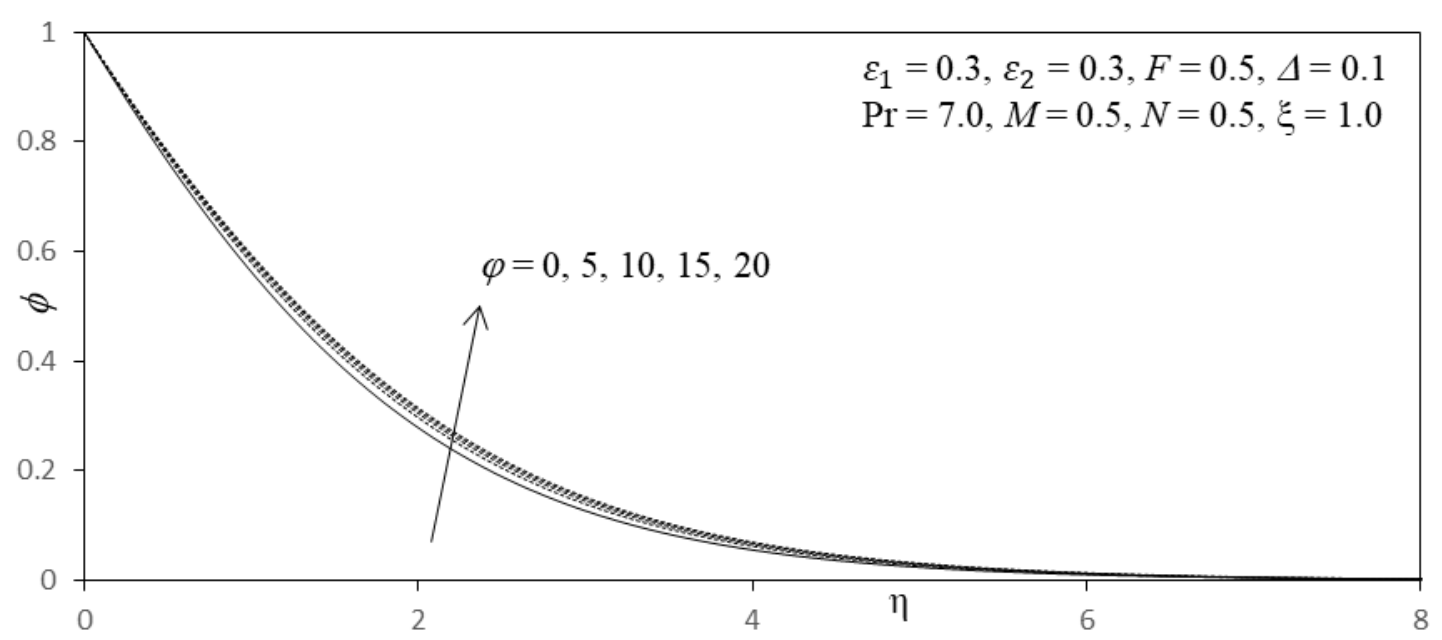

Fig.2(c) Effect of $\varphi$ on Concentration Profiles

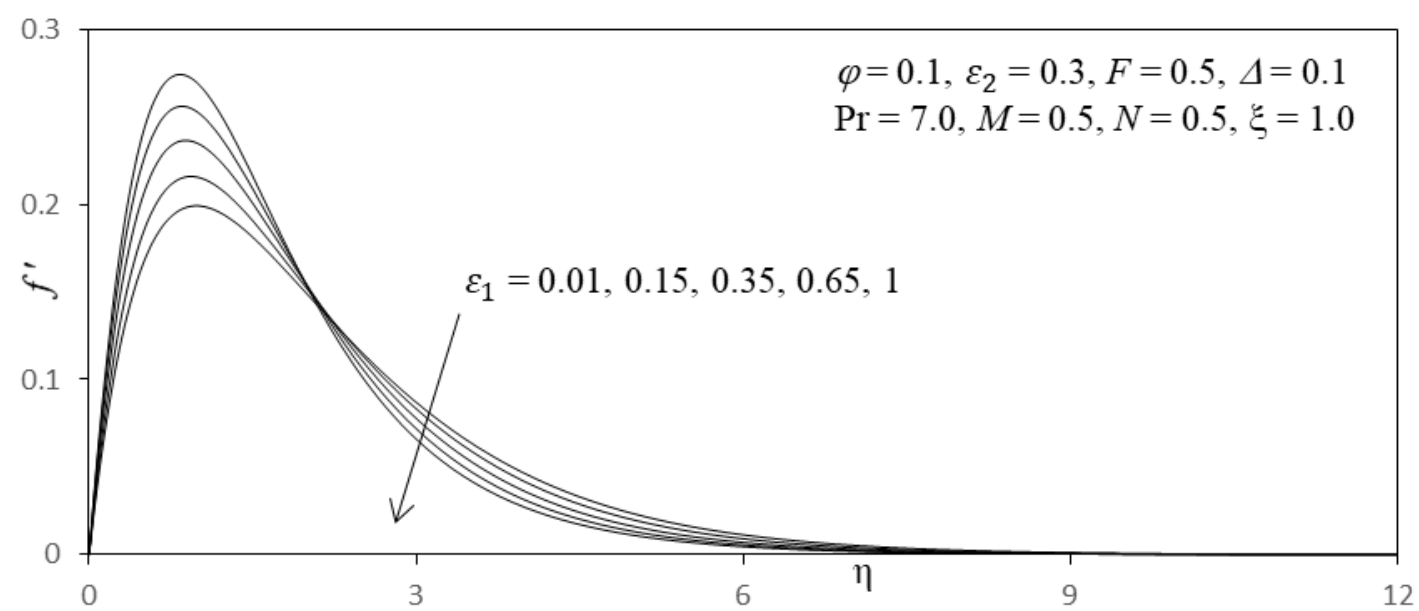

Fig.3(a) Effect of $\varepsilon 1$ on Velocity Profiles 


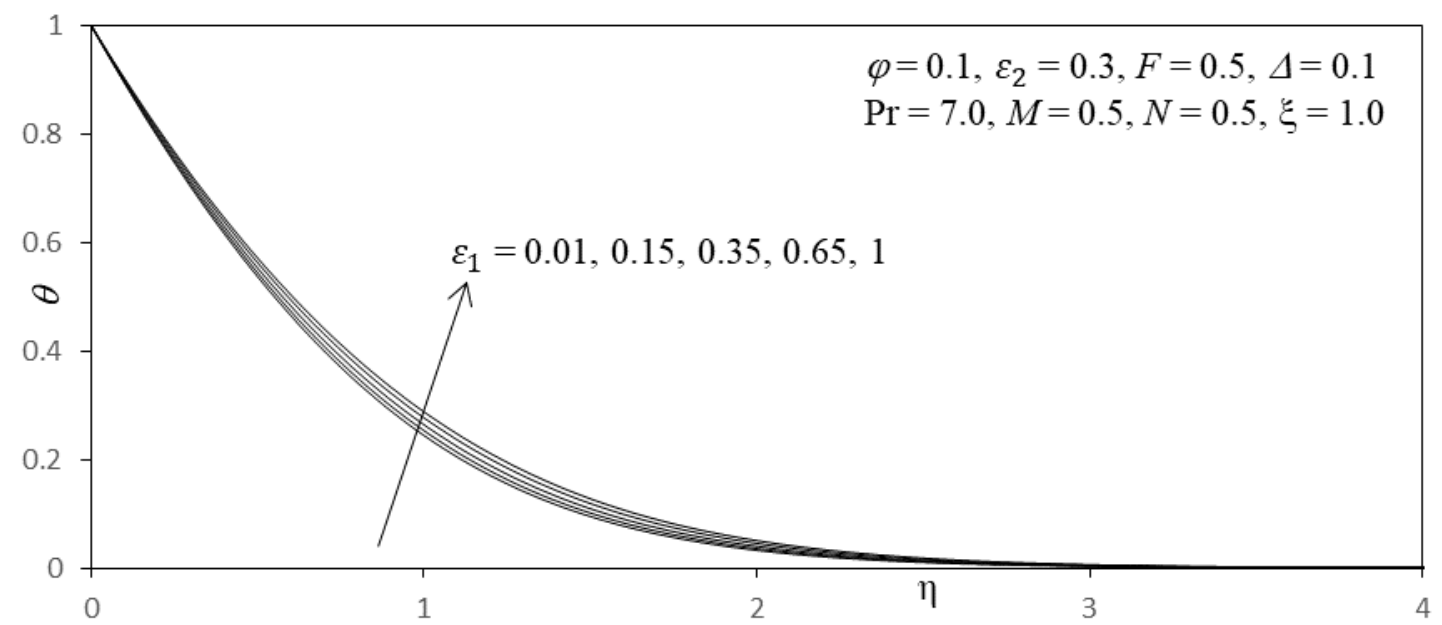

Fig.3(b) Effect of $\varepsilon_{1}$ on Temperature Profiles

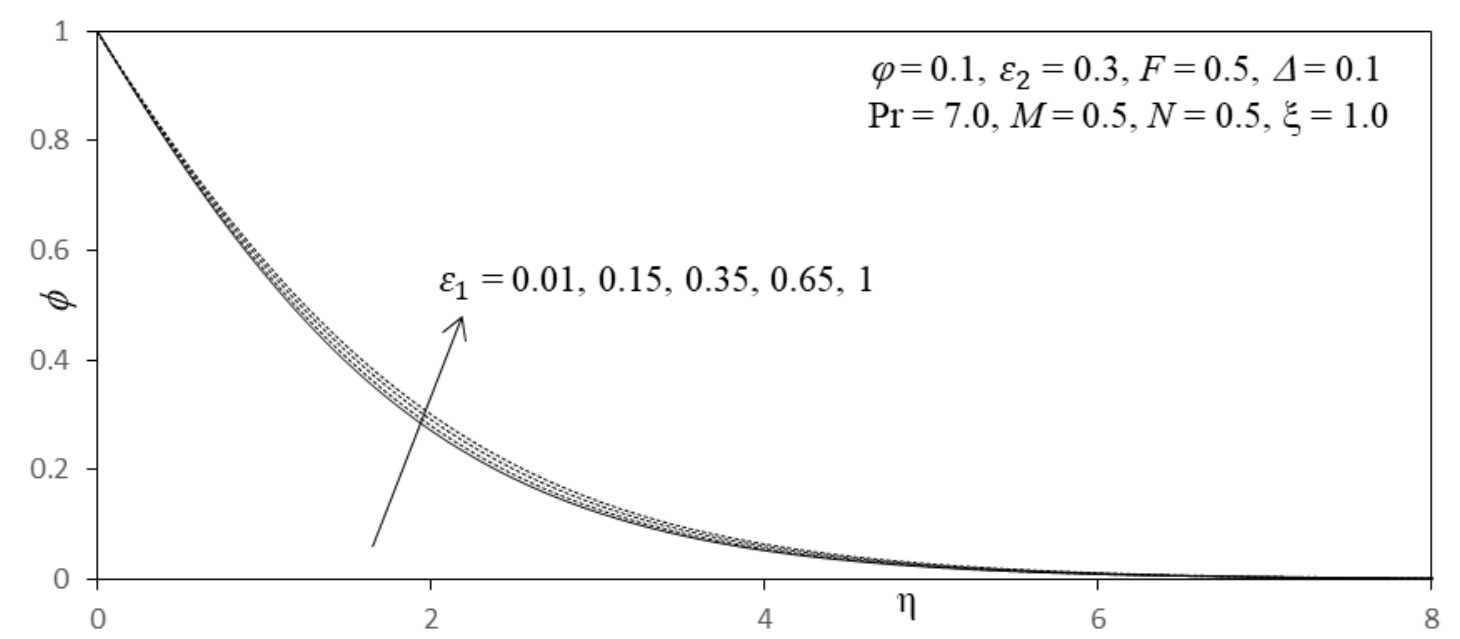

Fig.3(c) Effect of of $\varepsilon_{1}$ on Concentration Profiles

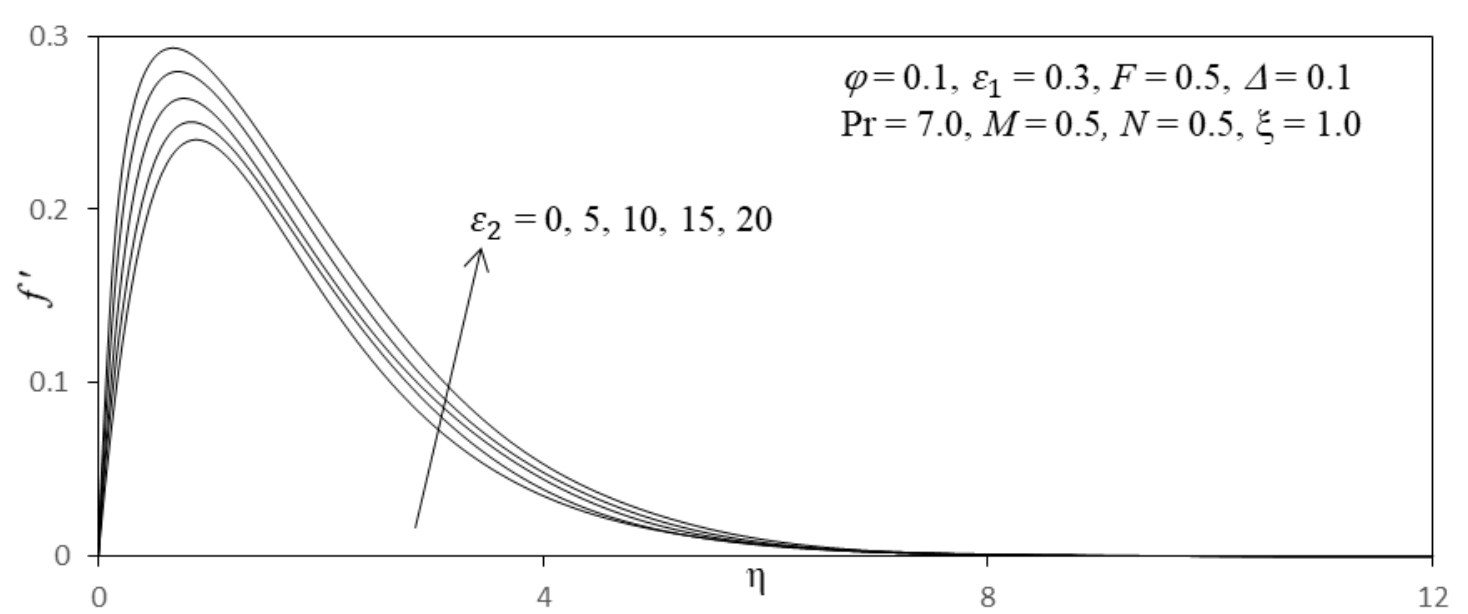

Fig.4(a) Effect of $\varepsilon_{2}$ on Velocity Profiles 


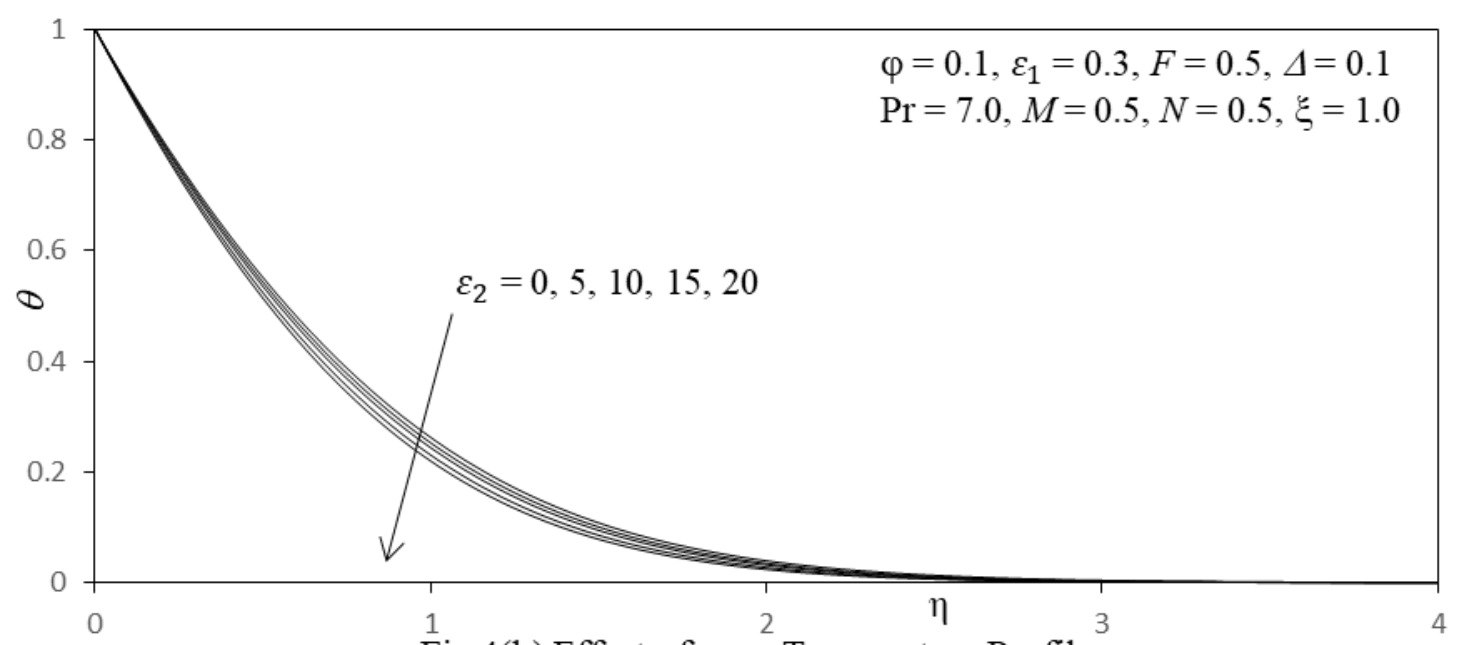

Fig.4(b) Effect of $\varepsilon_{2}$ on Temperature Profiles

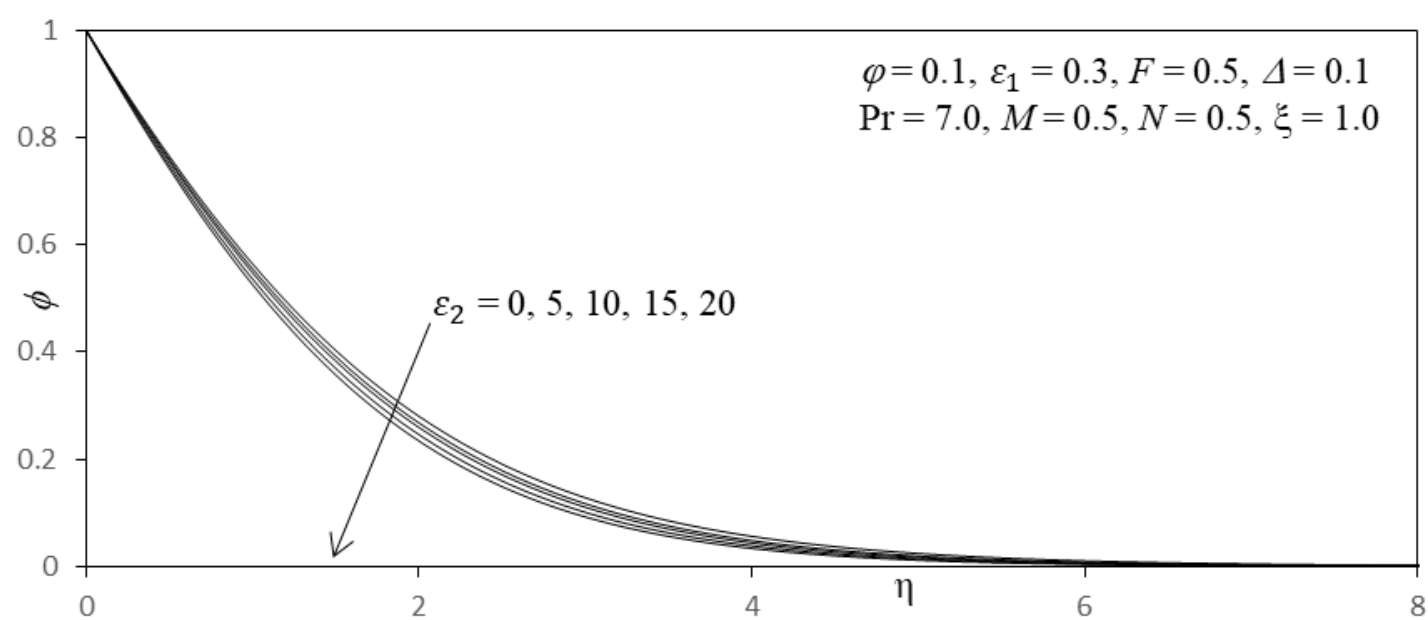

Fig.4(c) Effect of $\varepsilon_{2}$ on Concentration Profiles

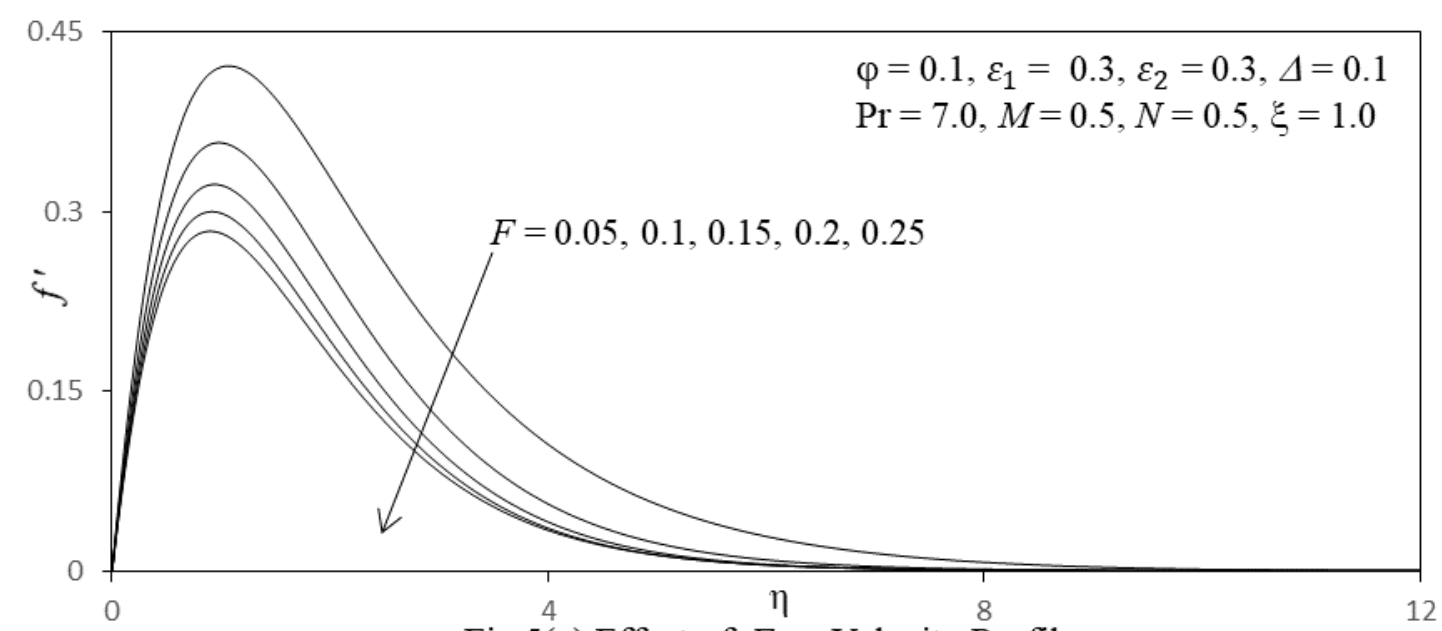

Fig.5(a) Effect of $F$ on Velocity Profiles 


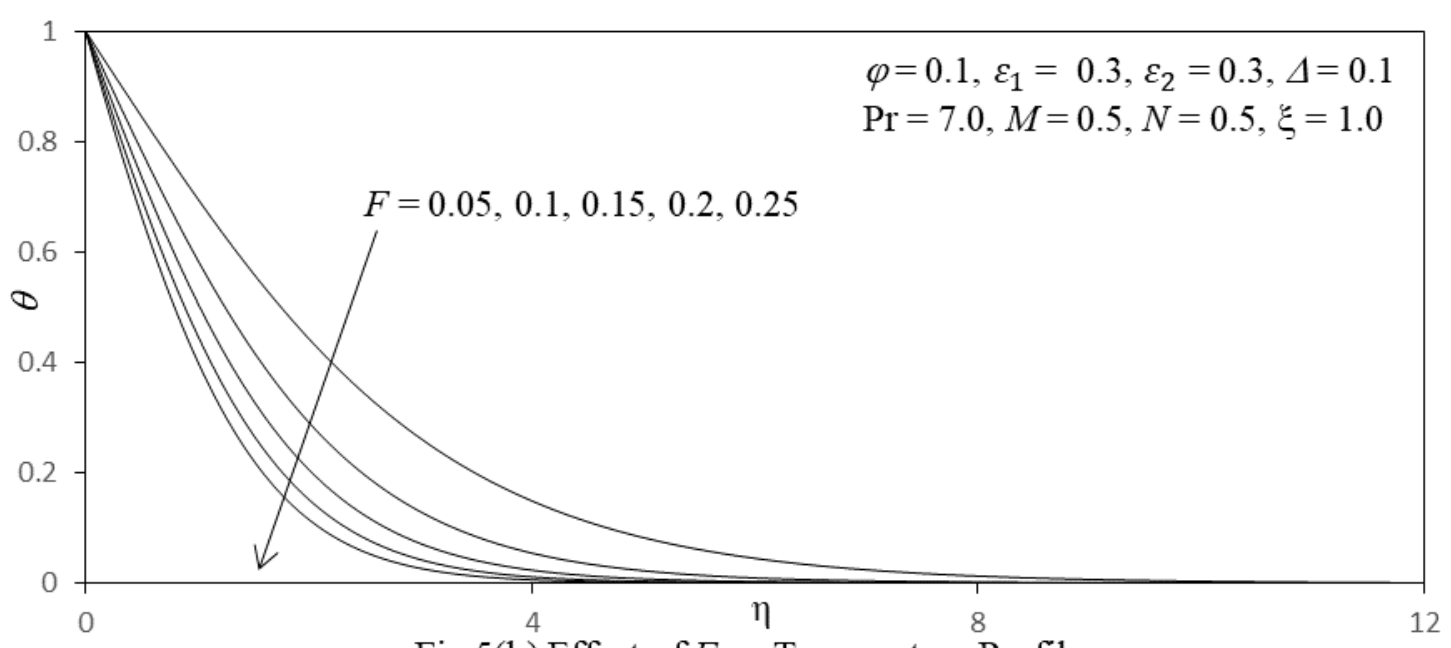

Fig.5(b) Effect of $F$ on Temperature Profiles

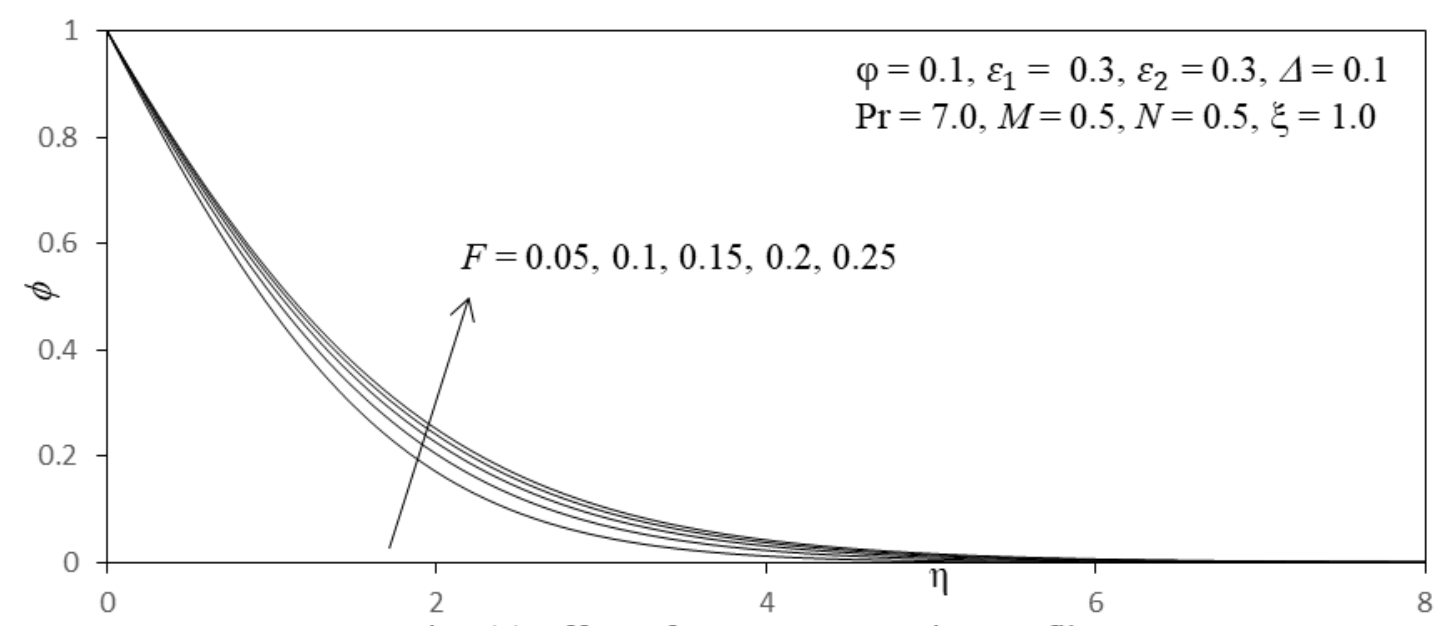

Fig.5(c) Effect of $F$ on Concentration Profiles

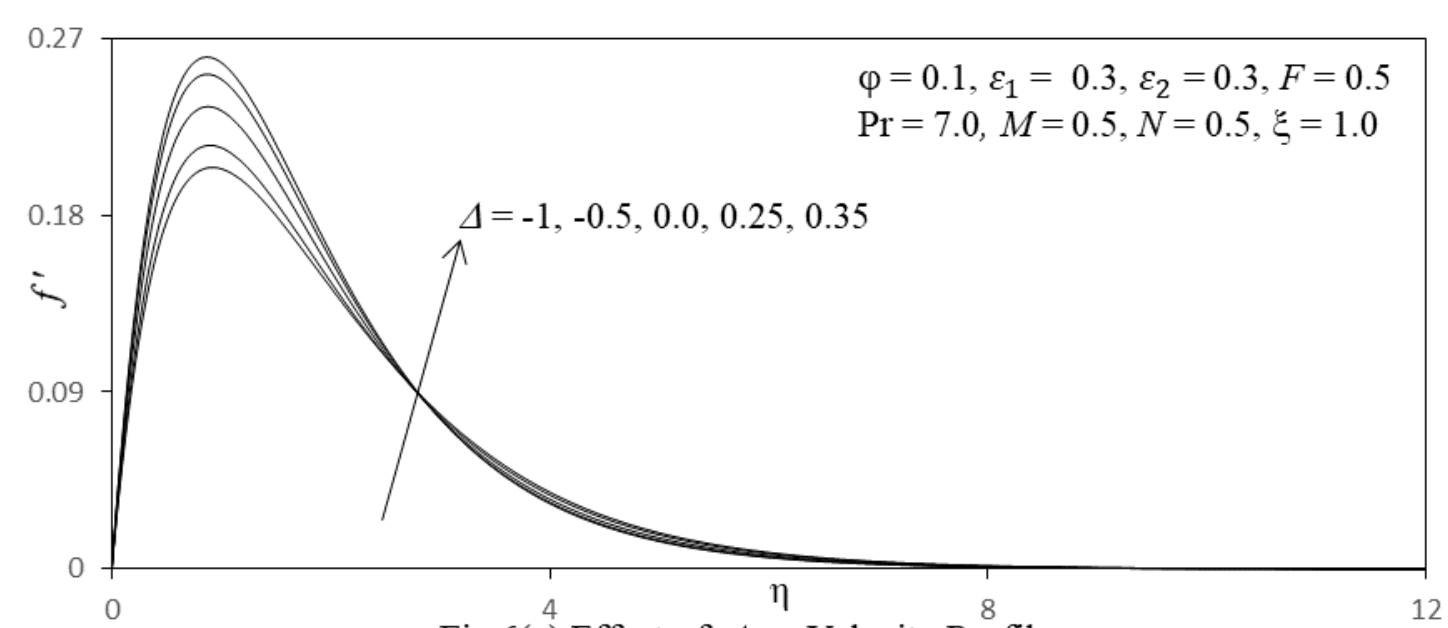

Fig.6(a) Effect of $\Delta$ on Velocity Profiles 


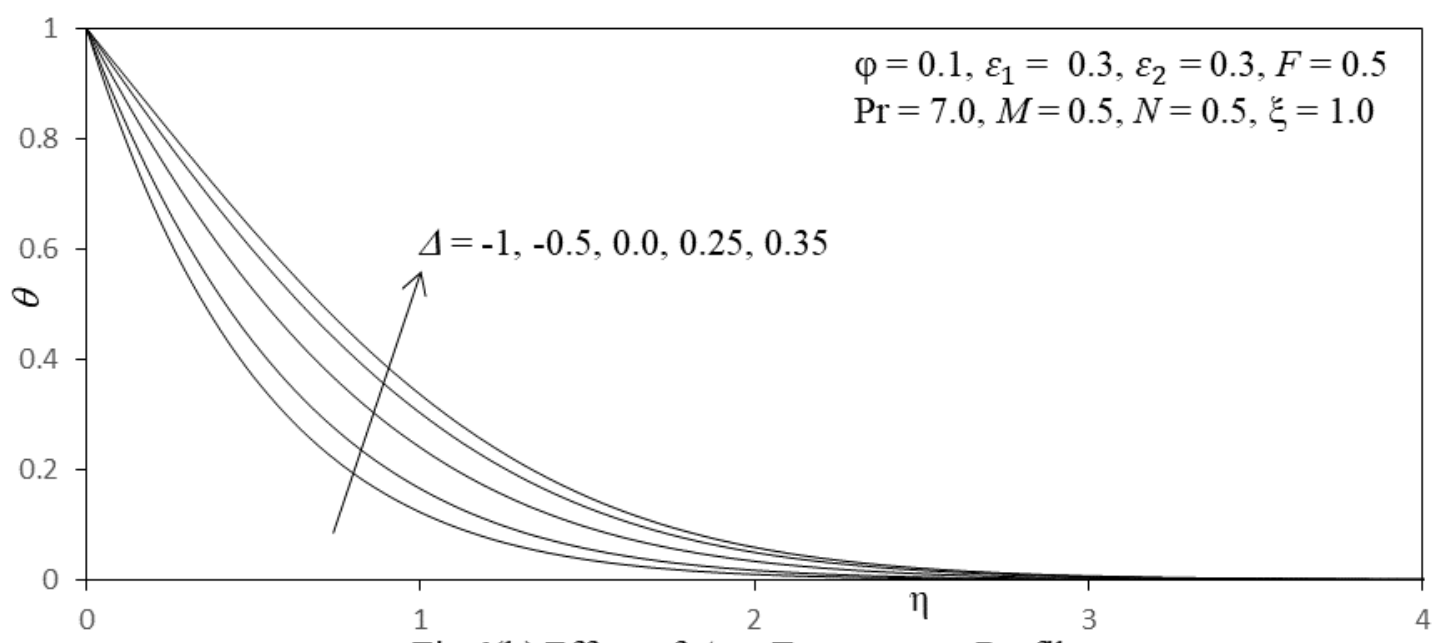

Fig.6(b) Effect of $\Delta$ on Temperature Profiles

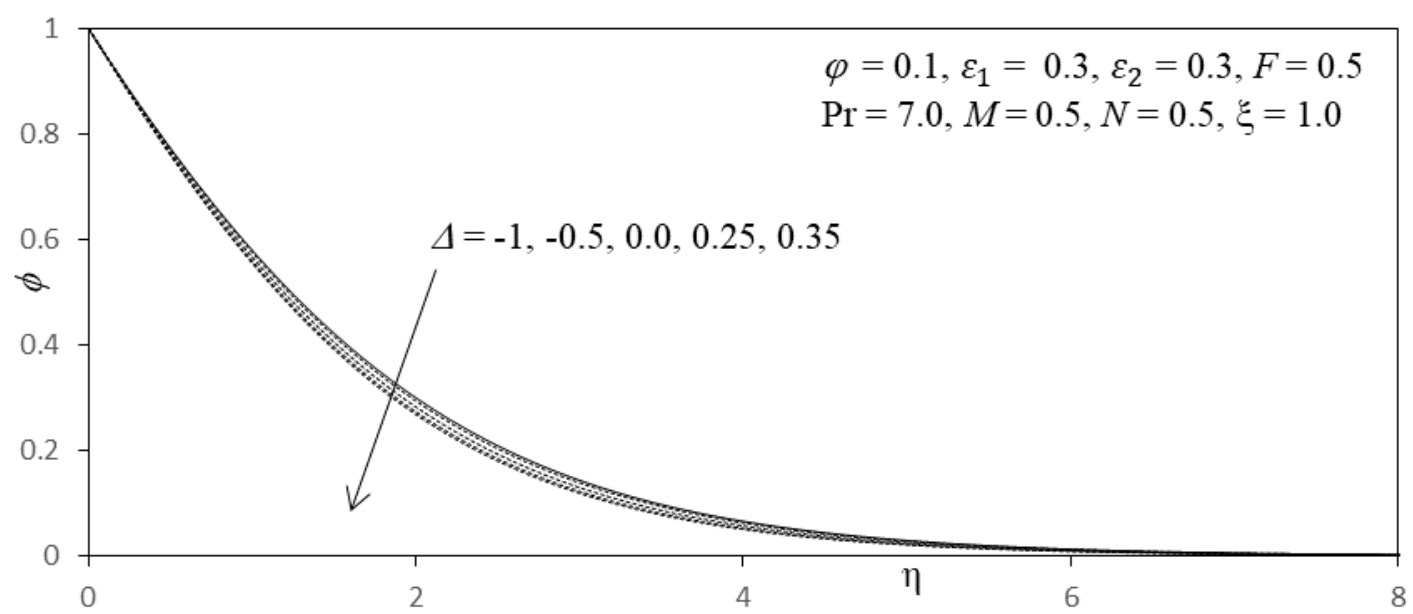

Fig.6(c) Effect of $\Delta$ on Concentration Profiles

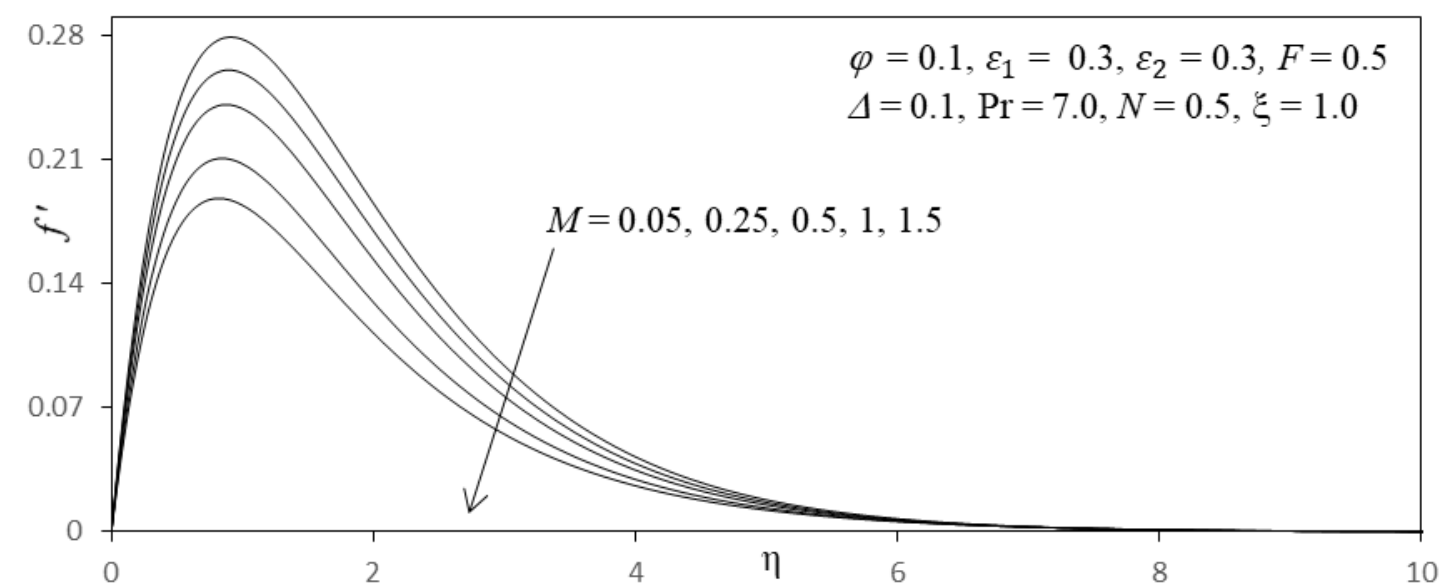

Fig.7(a) Effect of $M$ on Velocity Profiles 


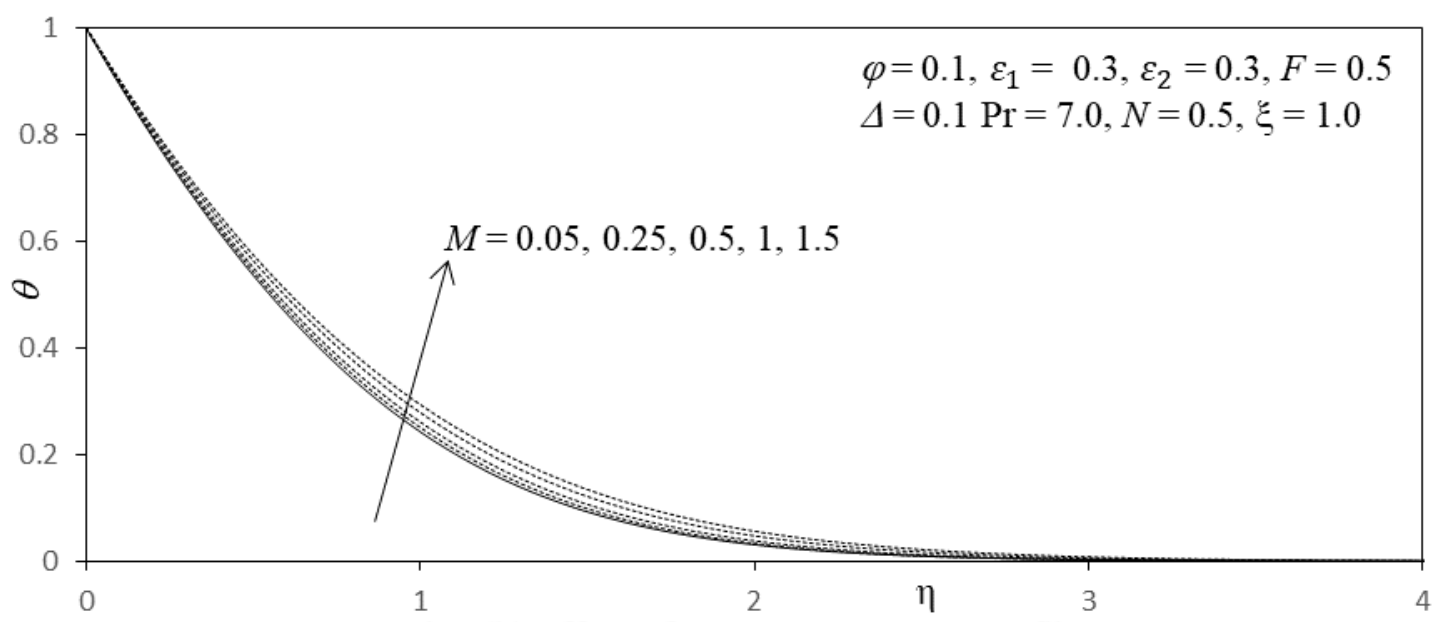

Fig.7(b) Effect of $M$ on Temperature Profiles

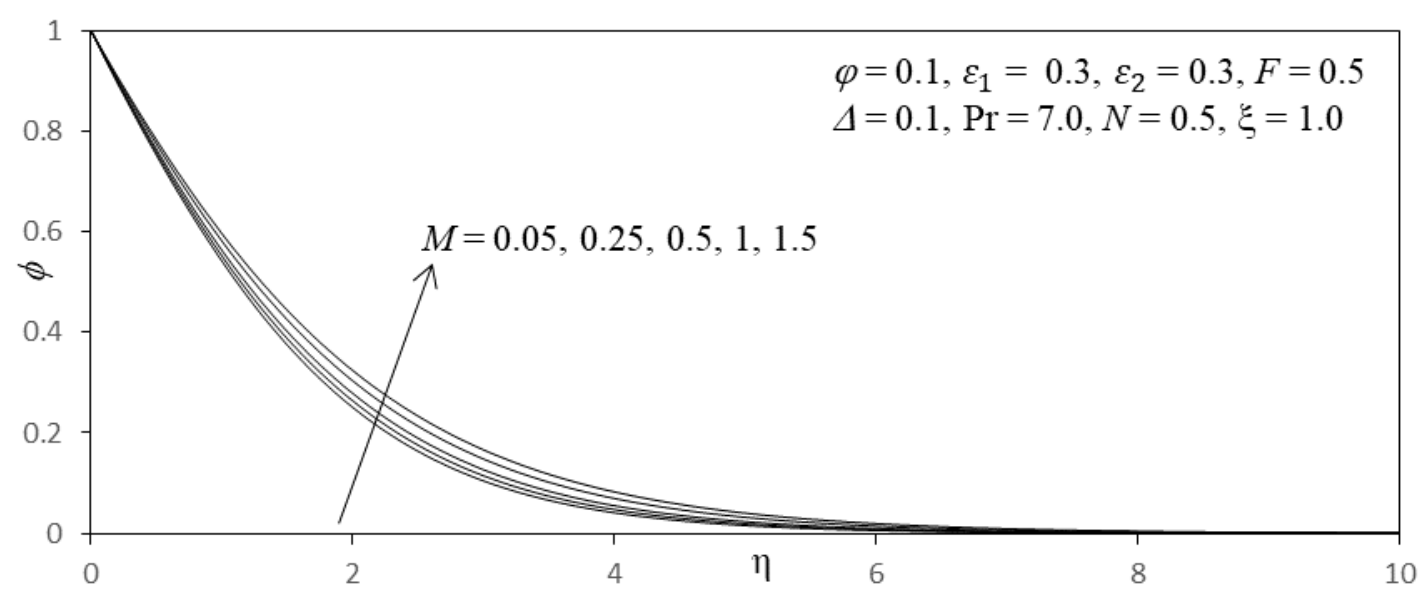

Fig.7(c) Effect of $M$ on Concentration Profiles

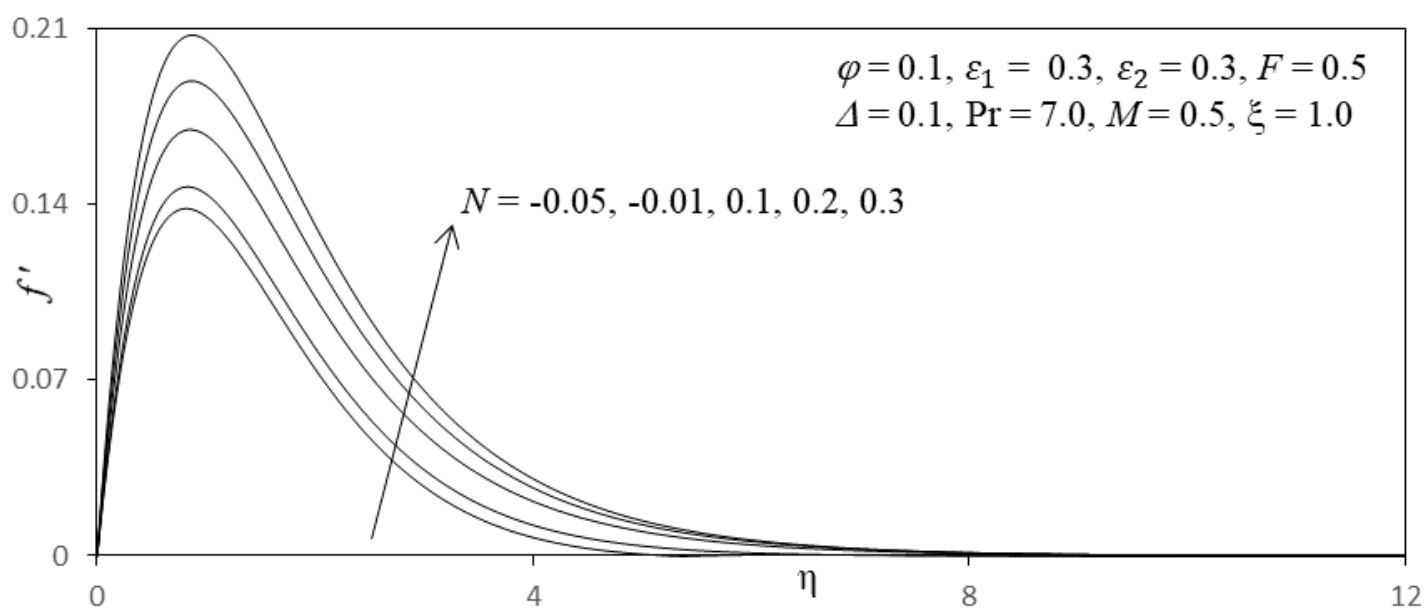

Fig.8(a) Effect of $N$ on Velocity Profiles 


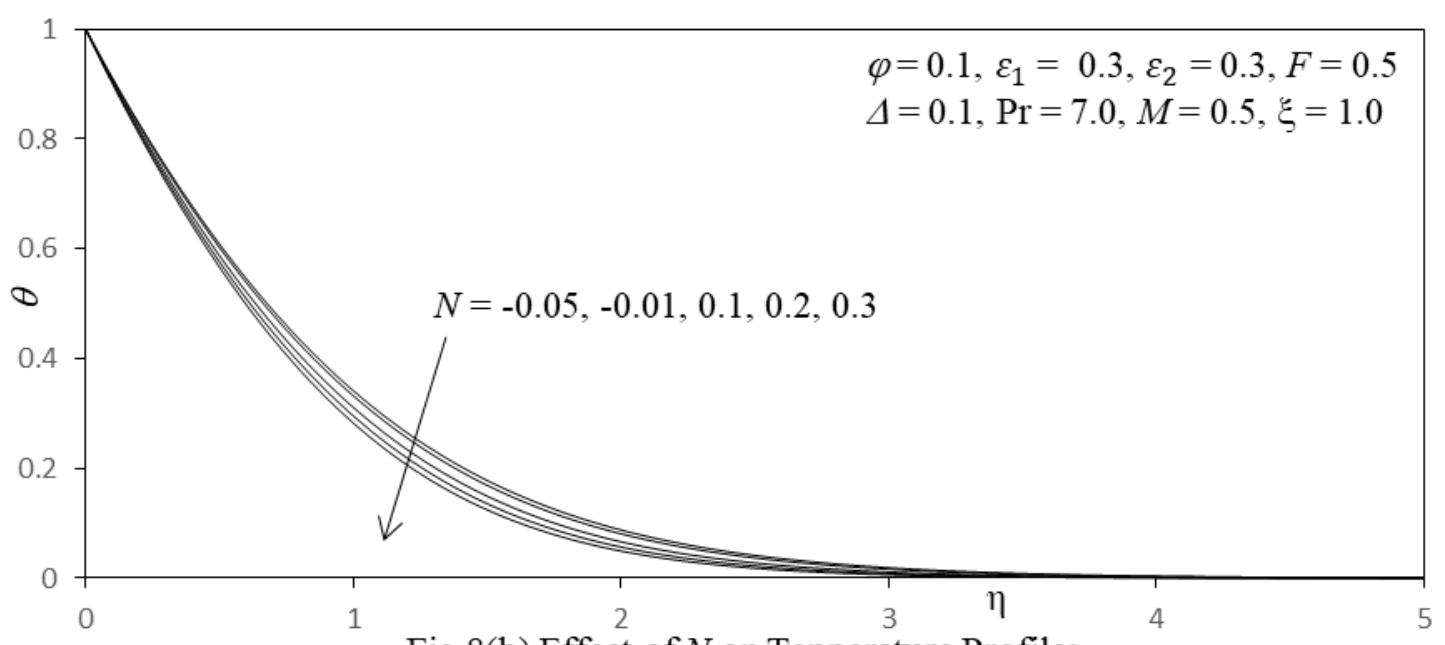

Fig.8(b) Effect of $N$ on Tenperature Profiles

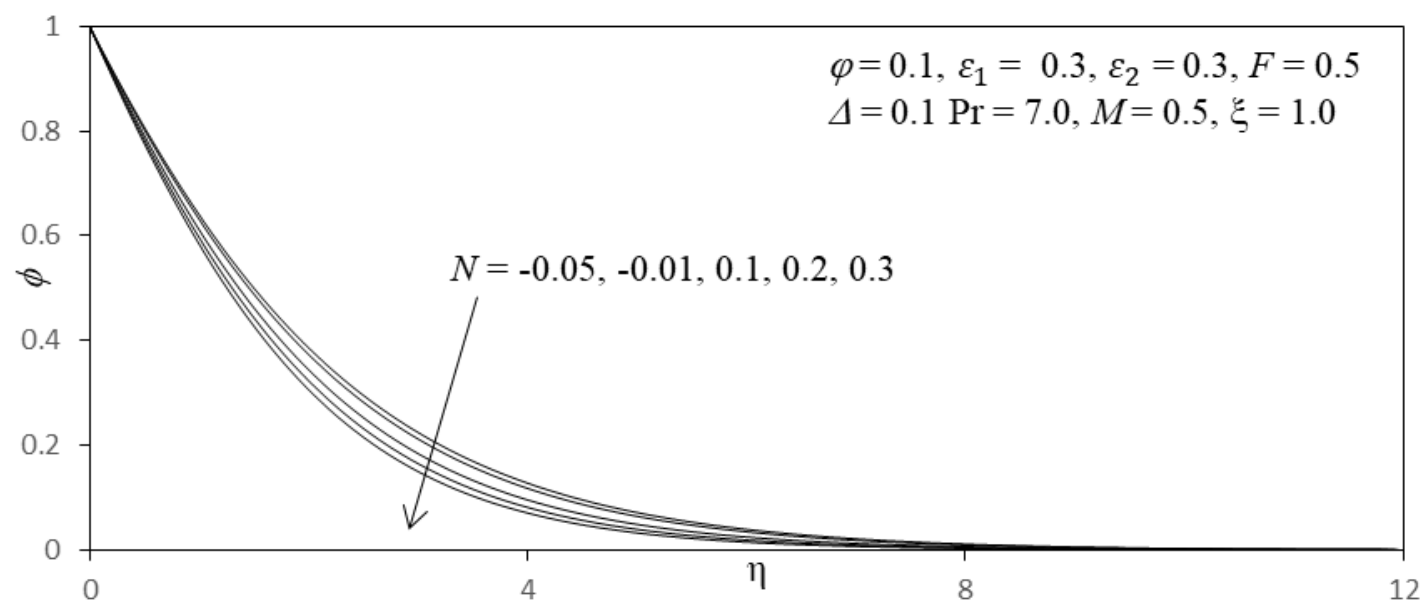

Fig.8(c) Effect of $N$ on Concentration Profiles

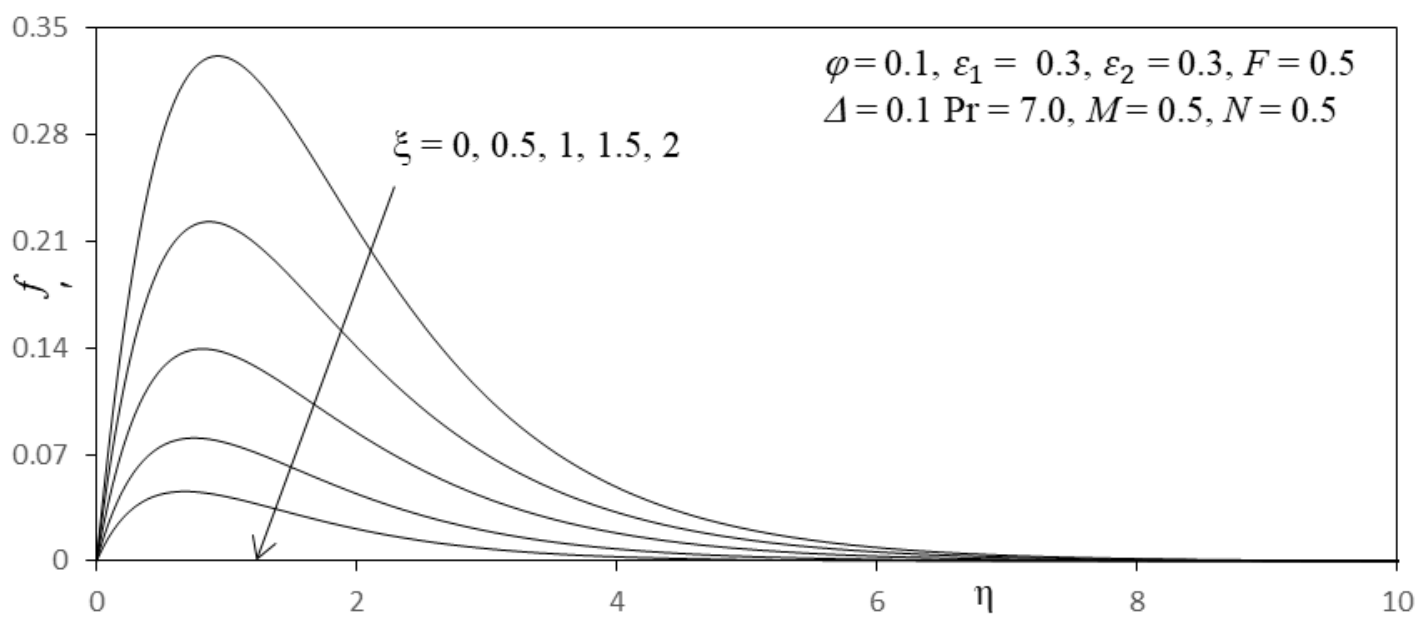

Fig.9(a) Effect of $\xi$ on Velocity Profiles 


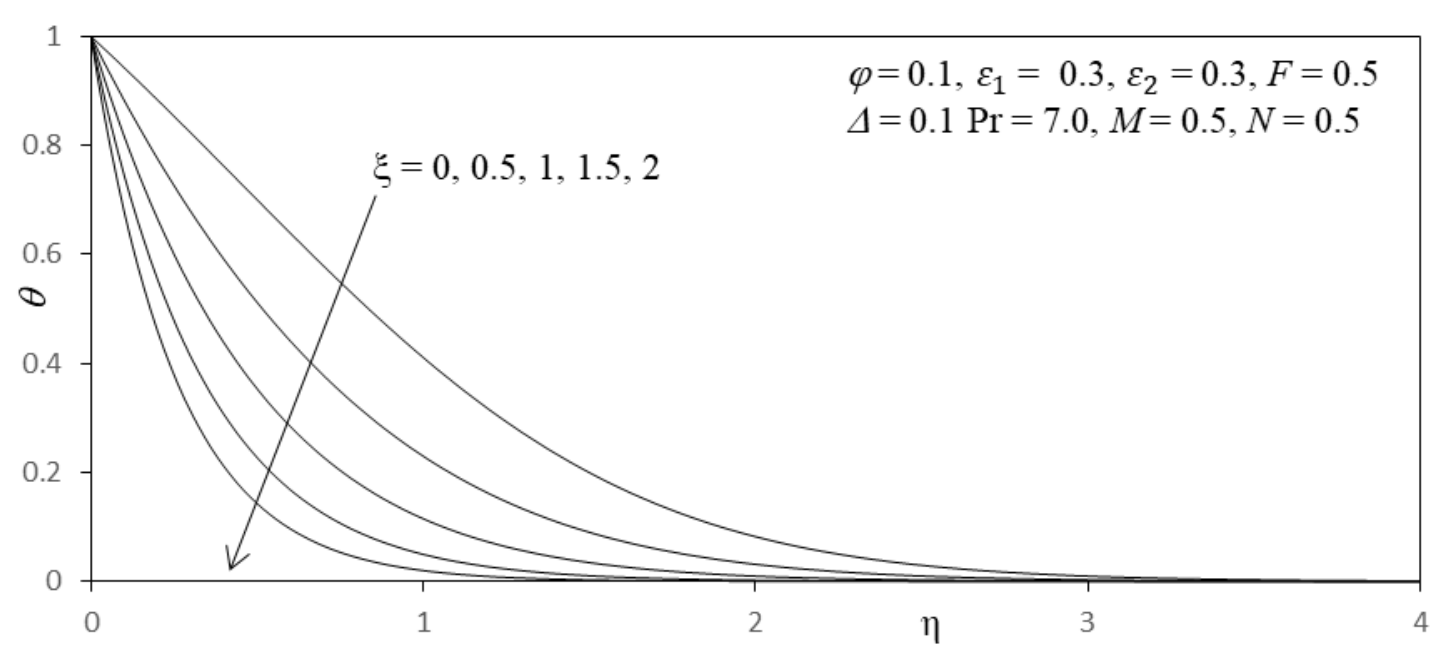

Fig.9(b) Effect of $\xi$ on Temperature Profiles

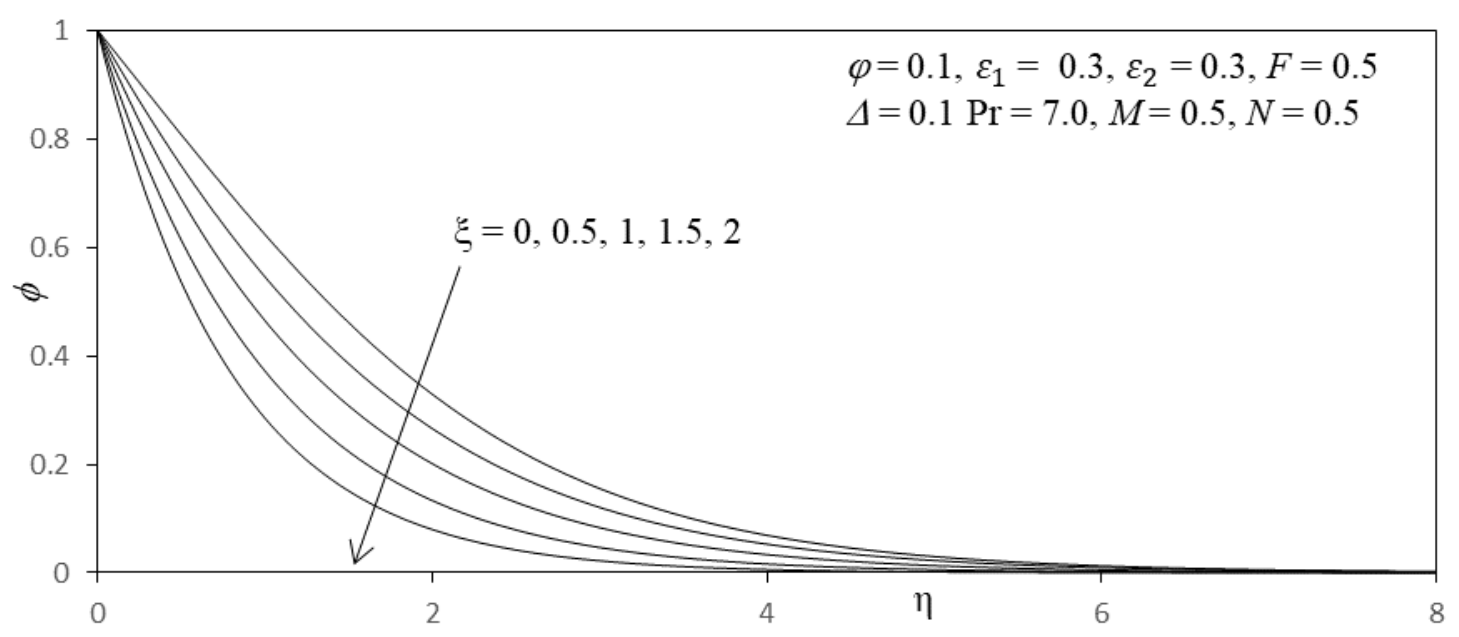

Fig.9(c) Effect of $\xi$ on Concentration Profiles

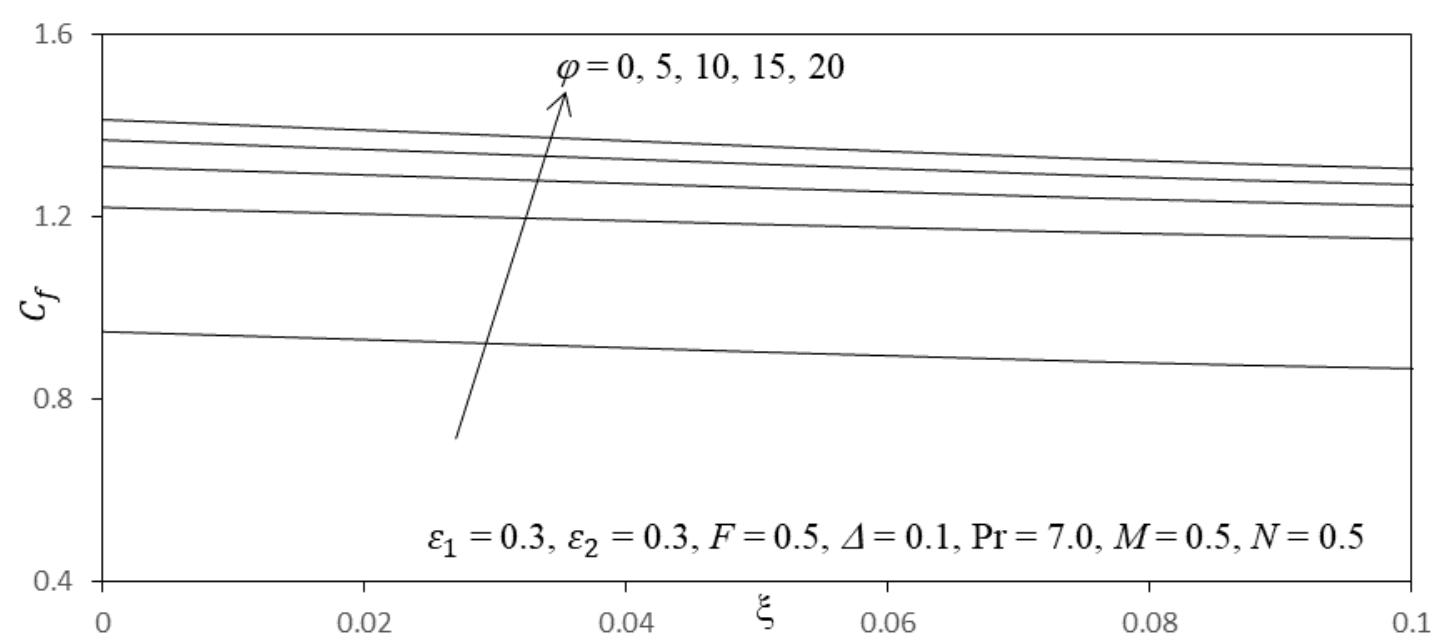

Fig.10(a) Effect of $\varphi$ on Skin Friction Coefficient 


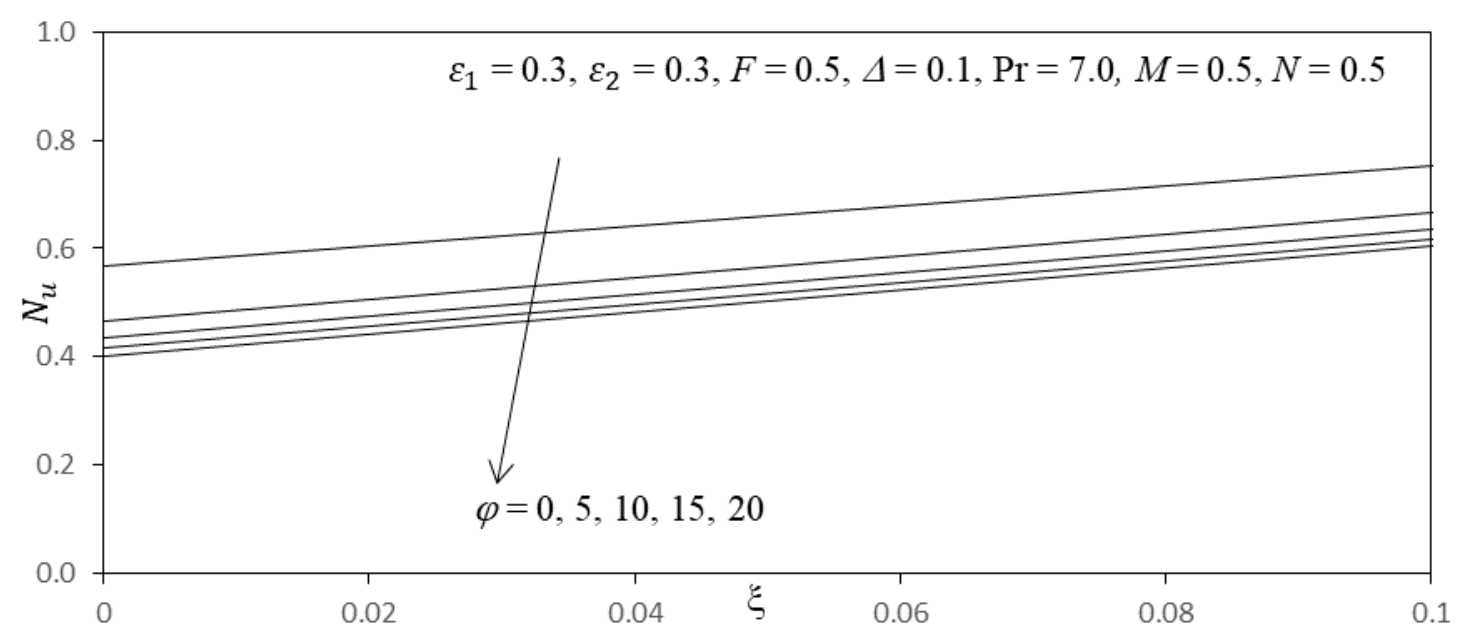

Fig.10(b) Effect of $\varphi$ on Local Nusselt number

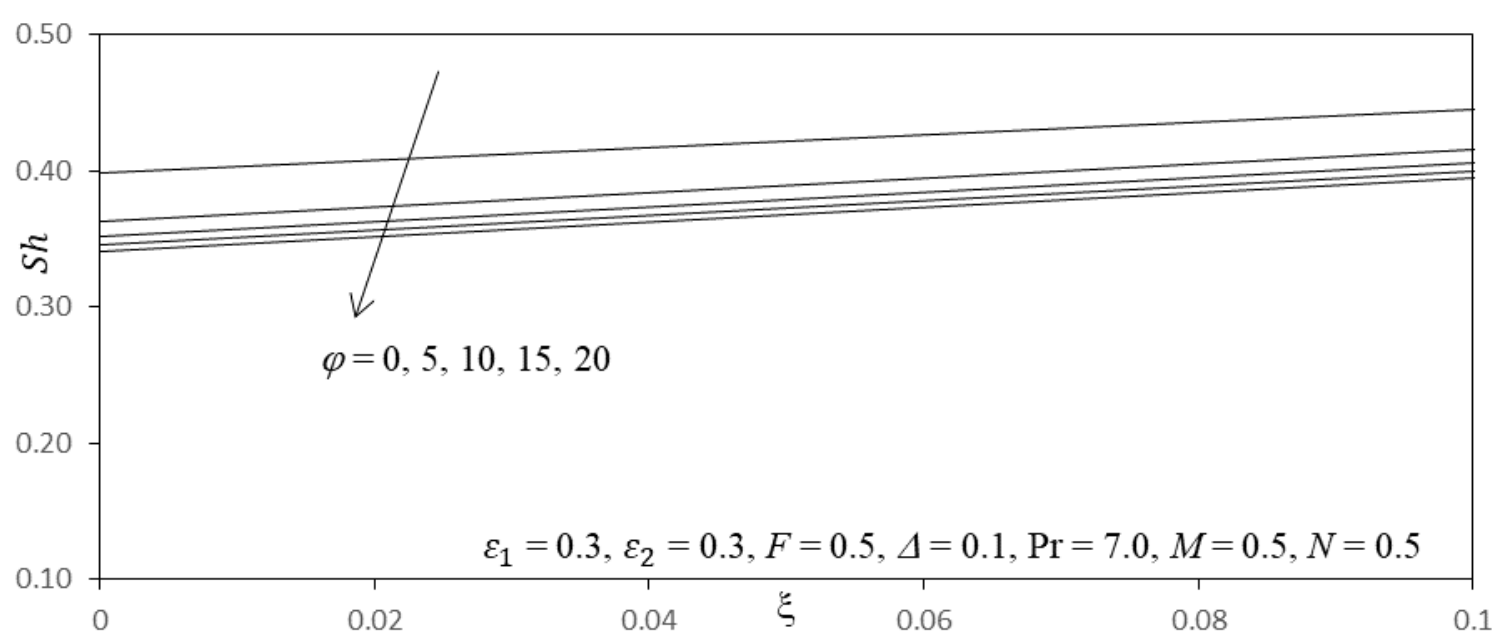

Fig.10(c) Effect of $\varphi$ on Local Sherwood Number

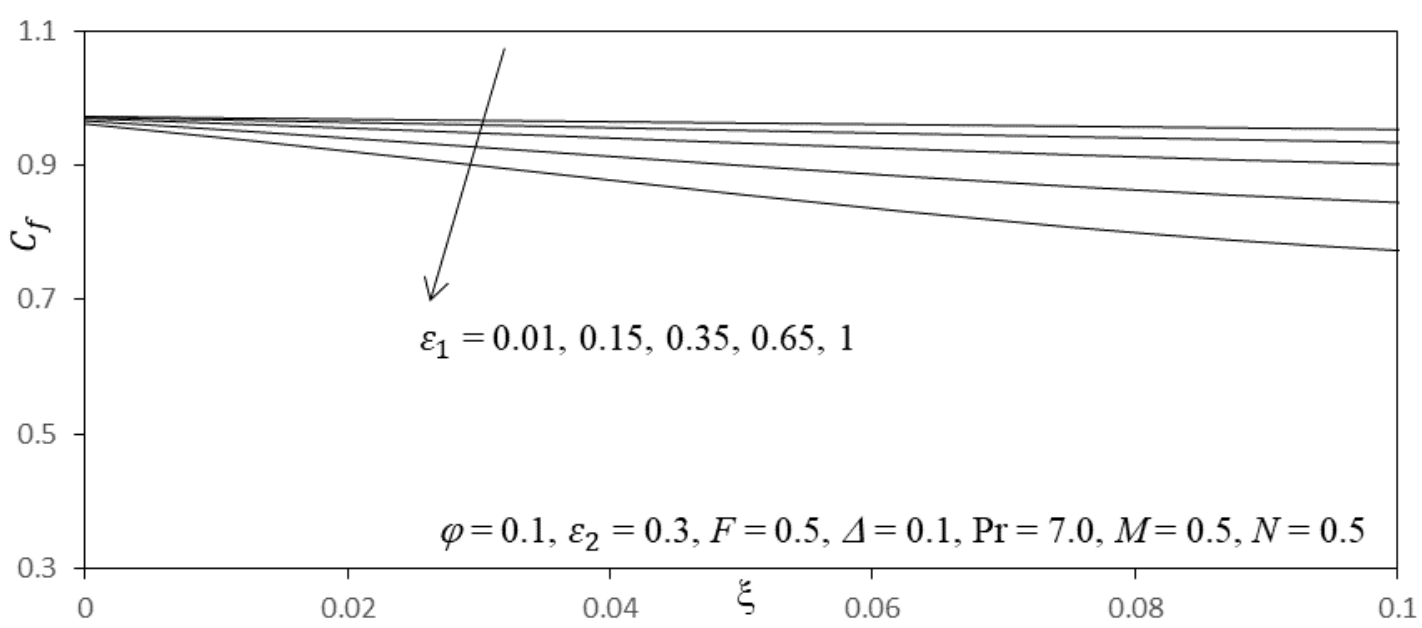

Fig.11(a) Effect of $\varepsilon_{l}$ on Skin Friction Coefficient 


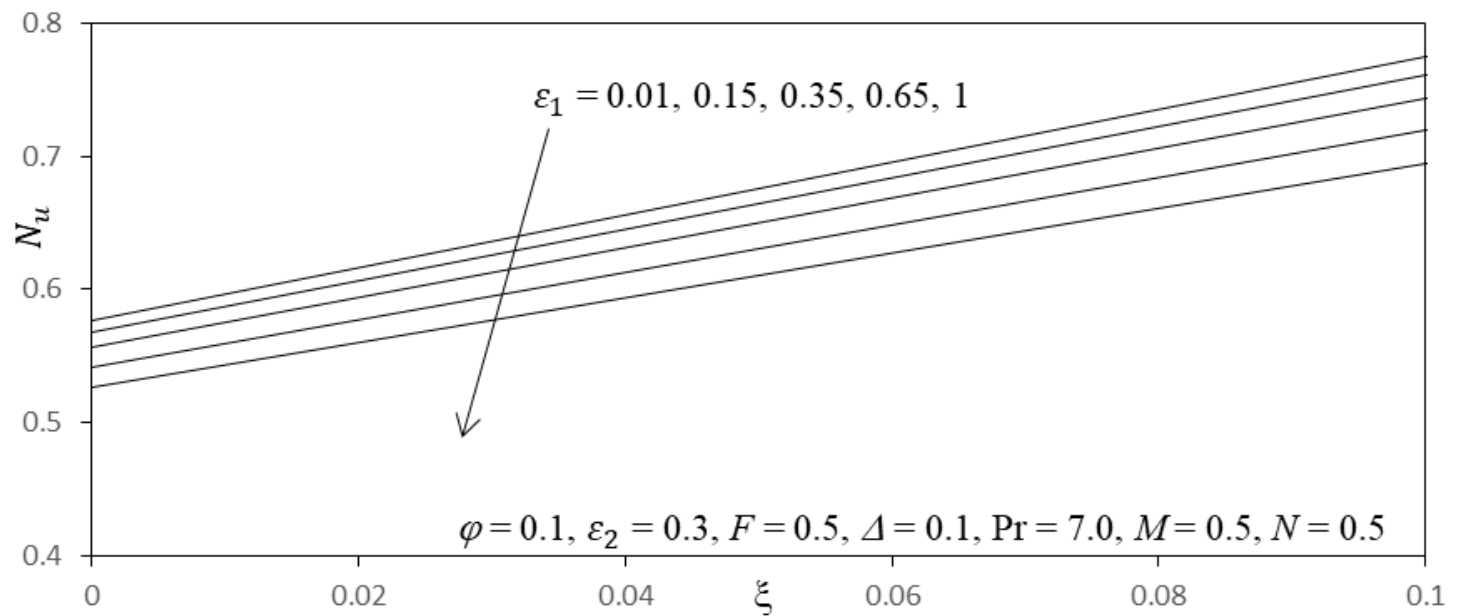

Fig.11(b) Effect of $\varepsilon_{l}$ on Local Nusselt number

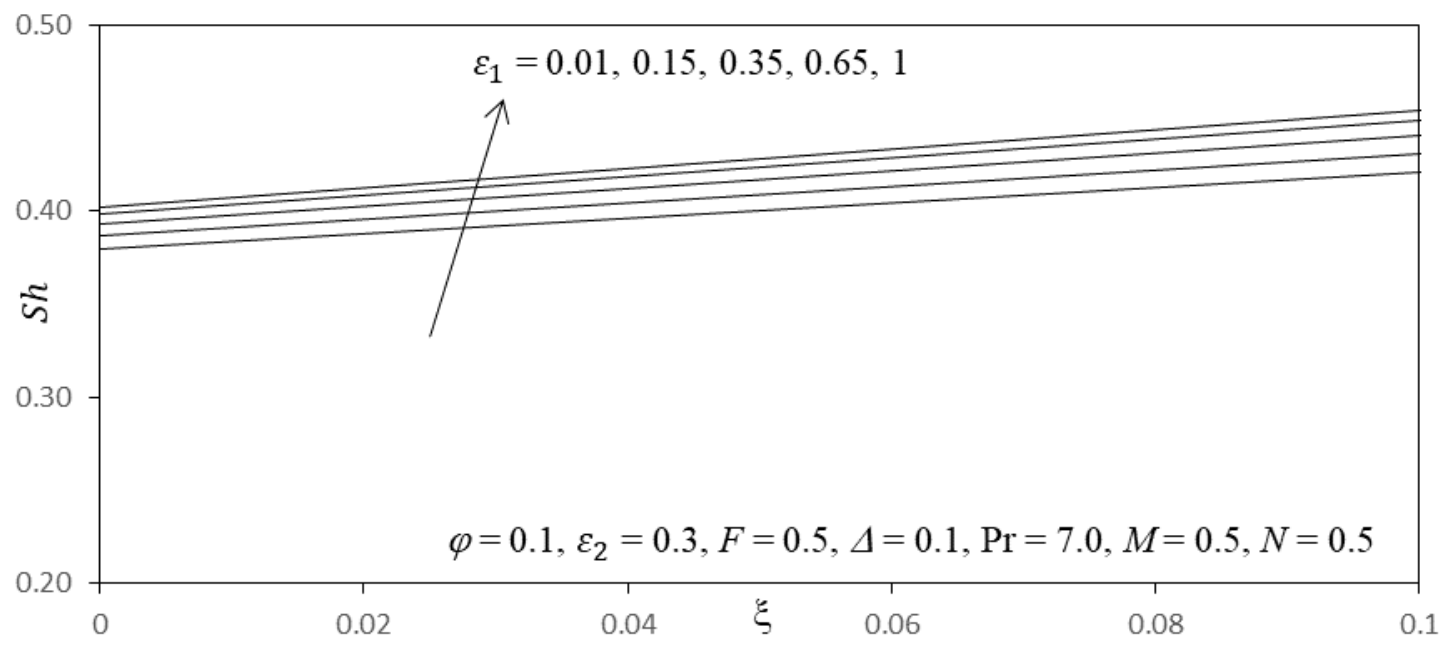

Fig.11(c) Effect of $\varepsilon_{l}$ on Local Sherwood Number 


\section{Tables}

Table 1: Comparison values of $-\theta^{\prime}(\xi, 0)$ for various values of $\xi$ with $\operatorname{Pr}=0.1, \varepsilon_{1}=\varepsilon_{2}=\phi=0$, $M=0, F=0.5, \Delta=0.1, S c=0.6, N=0.5$ for only isothermal, solid cone case selected from Hossain and Paul [24].

\begin{tabular}{|c|c|c|}
\hline \multirow{2}{*}{$\xi$} & \multicolumn{2}{|c|}{$-\theta^{\prime}(\xi, 0)$} \\
\cline { 2 - 3 } & Hossain and Paul [24] & Present results \\
\hline 0.0 & 0.24584 & 0.24583 \\
\hline 0.1 & 0.25089 & 0.25088 \\
\hline 0.2 & 0.25601 & 0.25599 \\
\hline 0.4 & 0.26630 & 0.26629 \\
\hline 0.6 & 0.27662 & 0.27658 \\
\hline 0.8 & 0.28694 & 0.28691 \\
\hline 1.0 & 0.29731 & 0.29729 \\
\hline 2.0 & 0.35131 & 0.35128 \\
\hline
\end{tabular}

Table 2: Values of $C_{f}, N u$ and $S h$ computed with KBM numerical approaches for different $N$, and $M$, $\xi$ with $\varepsilon_{1}=\varepsilon_{2}=0.3, \varphi=0.1, \operatorname{Pr}=7.0, F=0.5, \Delta=0.1$, and $S c=0.6$.

\begin{tabular}{|c|c|c|c|c|c|c|c|c|c|c|}
\hline \multirow{2}{*}{$M$} & \multirow{2}{*}{$N$} & \multicolumn{3}{|c|}{$\xi=1.0$} & \multicolumn{3}{|c|}{$\xi=2.0$} & \multicolumn{3}{|c|}{$\xi=3.0$} \\
\hline & & $C_{f}$ & $N_{u}$ & $S h$ & $C_{f}$ & $N_{u}$ & $S h$ & $C_{f}$ & $N_{u}$ & $S h$ \\
\hline 0.05 & \multirow{5}{*}{0.5} & 0.4177 & 1.6225 & 0.8942 & 0.1564 & 3.6671 & 1.3444 & 0.0738 & 5.6613 & 1.8754 \\
\hline 0.25 & & 0 & 1.6207 & 0.8937 & 1545 & 3.6668 & 1.3443 & 0.0735 & 5.6607 & 1.8753 \\
\hline 0.5 & & 0.3966 & 1.6186 & 0.8932 & .1523 & 3.6667 & 1.3442 & 0.0730 & 5.6570 & 1.8752 \\
\hline 1.0 & & 0.3762 & 1.6150 & 0.8922 & .1481 & 3.6666 & 1.3442 & 0.0720 & 5.6562 & 1.8751 \\
\hline 1.5 & & 0.3585 & 1.6122 & 0.8914 & .1443 & 3.6665 & 1.3440 & 0.0711 & 5.6596 & 1.8749 \\
\hline \multirow{5}{*}{0.5} & -0.05 & 0.2129 & 1.5731 & 0.8806 & 0.0512 & 3.6489 & 1.3337 & 0.0166 & 5.6394 & 1.8590 \\
\hline & -0.01 & 0.2262 & 1.5766 & 0.8816 & 0.0586 & 3.6508 & 1.3345 & 0.0207 & 5.6426 & 1.8601 \\
\hline & 0.1 & 0.2630 & 1.5860 & 0.8842 & 0.0788 & 3.6559 & 1.3366 & 0.0319 & 5.6497 & 1.8638 \\
\hline & 0.2 & 0.2963 & 1.5944 & 0.8865 & 0.0972 & 3.6591 & 1.3388 & 0.0422 & 5.6543 & 1.8673 \\
\hline & 0.3 & 0.3297 & 1.6026 & 0.8888 & 0.1156 & 3.6621 & 1.3408 & 0.0525 & 5.6573 & 1.8704 \\
\hline
\end{tabular}


Table 3: Values of $C_{f}, N u$ and $S h$ computed with KBM numerical approaches for different $F, \Delta$ and $\xi$ with $\varepsilon_{1}=0.3, \varepsilon_{2}=0.3, \varphi=0.1, \operatorname{Pr}=7.0, M=0.5, N=0.5$ and $S c=0.6$.

\begin{tabular}{|c|c|c|c|c|c|c|c|c|c|c|}
\hline \multirow{2}{*}{$F$} & \multirow{2}{*}{$\Delta$} & \multicolumn{3}{|c|}{$\xi=1.0$} & \multicolumn{3}{|c|}{$\xi=2.0$} & \multicolumn{3}{|c|}{$\xi=3.0$} \\
\hline & & $C_{f}$ & $N_{u}$ & $S h$ & $C_{f}$ & $N_{u}$ & $S h$ & $C_{f}$ & $N_{u}$ & $S h$ \\
\hline 0.05 & \multirow{5}{*}{0.1} & 0.9754 & 0.4147 & 0.8315 & 0.6469 & 0.5780 & 1.3045 & 0.3996 & 0.7826 & 1.8541 \\
\hline 0.05 & & 0.8299 & 0.6282 & 0.8008 & 0.4615 & 1.0056 & 1.2752 & 0.2348 & 1.4675 & 1.8392 \\
\hline 0.15 & & 0.7337 & 0.8319 & 0.7798 & 0.3612 & 1.4333 & 1.2606 & 0.1680 & 2.1304 & 1.8348 \\
\hline 0.2 & & 0.6658 & 1.0260 & 0.7652 & 0.3014 & 1.8739 & 1.2535 & 0.1357 & 2.7536 & 1.8330 \\
\hline 0.25 & & 0.6156 & 1.2106 & 0.7548 & 0.2648 & 2.2326 & 1.2498 & 0.1176 & 3.3384 & 1.8321 \\
\hline \multirow{5}{*}{0.5} & -1.0 & 0.4288 & 2.7267 & 0.7248 & 0.1862 & 4.5445 & 1.2444 & 0.0858 & 6.1259 & 1.8307 \\
\hline & -0.5 & 0.4481 & 2.4401 & 0.7269 & 0.1895 & 4.3416 & 1.2444 & 0.0859 & 5.9765 & 1.8308 \\
\hline & 0.0 & 0.4776 & 2.0882 & 0.7304 & 0.1934 & 4.1187 & 1.2445 & 0.0860 & 5.8196 & 1.8311 \\
\hline & 0.25 & 0.4999 & 1.8688 & 0.7331 & 0.1956 & 3.9976 & 1.2445 & 0.0861 & 5.7380 & 1.8316 \\
\hline & 0.35 & 0.5115 & 1.7677 & 0.7345 & 0.1965 & 3.9469 & 1.2446 & 0.0862 & 5.7049 & 1.8319 \\
\hline
\end{tabular}

